\title{
Effekte der diskursiven Verknüpfung von Antifeminismus und Rassismus
}

\section{Eine Fallstudie zu Orientierungskursen für neu Zugewanderte}

\author{
Denise Bergold-CALDWELl \& BARBARA GRUBNER \\ UNTER MitARbEIT VON PATRICIA RAMS
}

\section{EinLEitung}

Ein auffälliges Merkmal der aktuell konstatierten „autoritären Wende“ (Opratko 2019: 141) bzw. des gesellschaftlichen „Rechtsrucks“ (Kopke 2017: 50) ist darin zu sehen, dass sich antifeministische Mobilisierungen zunehmend mit rassistischen Denkfiguren verknüpfen. Dieser diskursiven Verknüpfung widmet sich unsere Fallstudie und stellt die Frage, wie gegenwärtig Artikulationen ausgestaltet sind, die sowohl gegen feministische Forderungen als auch gegen Zuwanderung mobilisieren. Damit richten wir unseren Blick auf jenes Phänomen, das in der feministischen Literatur als „Ethnisierung von Sexismus“ (Jäger 1999) bzw. „Ethnosexismus“ (Dietze 2017) bezeichnet wird - die Skandalisierung von Sexismus, geschlechtsbezogener Gewalt, Frauen- und Homosexualitätsfeindlichkeit als Sonderprobleme ,kulturell Anderer ${ }^{\varsigma}$ - und fragen danach, wie sich diese Form der Ethnisierung mit Einsprüchen gegen Feminismus und Gleichstellung verbindet. Dass es sich dabei nur an der Oberfläche um eine paradoxe Verknüpfung handelt, die zudem historisch keineswegs neu ist, macht Leila Ahmeds Hinweis auf die Doppelstrategie der britischen Kolonialherrschaft deutlich:

„[D]as männliche viktorianische Establishment suchte nach Strategien, mit denen es im eigenen Land feministische Forderungen abwehren konnte: Die Ideen des Feminismus wurden lächerlich gemacht und die Vorstellung, dass Männer Frauen unterdrücken, verworfen. Doch im Dienst des Kolonialismus griff dieses Establishment die Sprache des Feminismus auf und richtete sie gegen ,andere Männer“ und deren Kultur.“ (Ahmed 1992: 151, zit. nach von Braun/Mathes 2007: 313) 
Zuletzt spielte die Ethnisierung von Sexismus in der sogenannten Multikulturalismus-Debatte der 1990er Jahre eine Rolle, in der frauenunterdrückende Geschlechternormen migrantischer Familien kontrovers diskutiert wurden. Geschlechterforscherinnen haben in diesem Zusammenhang darauf hingewiesen, dass die besondere Betonung der - kulturell begründeten - Schlechterstellung von migrantischen Frauen herrschaftskritisch zu durchleuchten ist (Jäger 1999; Gutiérrez Rodríguez 1997; Strasser 2010). Feministische Forderungen, so wird an beiden Beispielen deutlich, können auch als Herrschaftsinstrumente in Dienst genommen werden nämlich dann, wenn bestimmte Personengruppen als Trägerschaft eines gesamtgesellschaftlichen Problems identifiziert werden und ungelöste Fragen der Geschlechterhierarchie (die Frauen nach wie vor global betrifft) und das Zusammenwirken verschiedener Herrschaftsformen (wie Sexismus und Rassismus) aus dem Blick rücken.

Heutige Verknüpfungen von Antifeminismus mit der Ethnisierung von Sexismus, müssen vor dem Hintergrund der gesellschaftspolitischen Debatte um die Einwanderung nach Europa seit 2015 und die Zunahme, Erweiterung und Vernetzung antifeministischer Diskurse und Akteur_innen ${ }^{1}$ der letzten Jahre analysiert werden. Im deutschsprachigen Raum sind es die Vorfälle in der Kölner Silvesternacht 2015/16, die diese Diskursproduktion besonders befeuert haben: In der Nacht vom 31.12.2015 zum 01.01.2016, so wissen wir heute durch polizeiliche Ermittlungen und Medienrecherchen, wurde eine große Zahl von Frauen auf der Kölner Domplatte Opfer von Einschüchterung, sexueller Belästigung und Diebstahl durch Männergruppen. Nur eine geringe Anzahl der über 600 Anzeigen sexueller Übergriffe wurde aufgeklärt und führte zu Verurteilungen. Dies lag zum einen an den nächtlichen Lichtverhältnissen und Turbulenzen der Silvesterfeier, an der Tausende Menschen teilnahmen, was die Ermittlungen erschwerte. Zum anderen lag dies an Lücken im Strafrecht. Die Einführung von zwei neuen Strafvorschriften gegen sexuelle Belästigung (§184i StGB) und gegen Gruppengewalt (§184j StGB) bei der Reform des Sexualstrafrechts im Juni 2016 gilt als direkte Reaktion auf die Übergriffe in der Kölner Silvesternacht (kritisch vgl. Hoven 2018).

In der öffentlichen Debatte nach Köln stand nicht das Phänomen Männergewalt im Zentrum. Es ging auch nicht darum, was sexualisierte und geschlechtsbezogene Formen der Gewalt mit den bestehenden Geschlechterverhältnissen zu tun haben. Vielmehr interessierte an den Ereignissen in Köln vor allem, dass die Täter mehrheitlich Migranten und Asylwerber waren. Diese Tatsache wurde von rechtspopulistischer Seite sehr schnell für migrationsfeindliche Hetze in Dienst genommen,

1 Wir verwenden für unseren Text die Schreibweise des Gender-Gaps, um deutlich zu machen, dass wir im Text alle Geschlechterpositionen ansprechen wollen, auch solche, die sich nicht in der Binarität von weiblich und männlich verorten. 
fand aber auch im bürgerlichen Feuilleton vorrangige Beachtung. Nicht für alle gegenwärtigen Positionen, die Antifeminismus und Rassismus miteinander verschränken, spielt Köln die Hauptrolle. Es lässt sich jedoch sagen, dass die Silvestervorfälle medial und gesellschaftspolitisch zu einer Diskursverschiebung in Bezug auf die Ethnisierung von Sexismus führten, die neue Anschlussmöglichkeiten an antifeministische Mobilisierungen bietet.

In der Darstellung unserer Forschungsergebnisse skizzieren wir im folgenden Abschnitt zunächst unser Forschungsdesign (Kap. 2). Danach stellen wir die von uns identifizierten Diskursformationen am Kreuzungspunkt antifeministischer und rassifizierender Denkfiguren vor (Kap. 3) und widmen uns schließlich den Effekten dieser Diskurse auf Orientierungskurse für neu Zugewanderte (Kap. 4). In unserer Fallstudie zeigte sich, dass offen antifeministische Diskurse dort keinen Platz finden. Worauf wir aber trafen, waren zum einen diskursive Zuspitzungen aus der Problematisierung von Köln, die zu Ethnisierungsdynamiken und zur pauschalen Heraushebung oder Adressierung bestimmter Personengruppen führten. Zum anderen wurde eine in Teilen postfeministische Art und Weise deutlich, Geschlecht und Sexualität zum Thema zu machen, während race als Kategorie dethematisiert wird oder hinter den Begriffen Kultur oder Diversität verschwindet. Im Fazit (Kap. 5) verdichten wir unsere Befunde zu vier Thesen: Zum einen schlagen wir vor, das Kursgeschehen (auch) als eine Form der Pädagogisierung (Kessl 2011) von Sexualität und Geschlecht in der gegenwärtigen Transformation des Wohlfahrtsstaates zu verstehen. Unsere zweite These ist, dass Gender- und Diversitätssensibilisierungen als postfeministische Instrumente zum Einsatz kommen können. Drittens problematisieren wir, dass die Teilnehmenden im Orientierungskurs vorrangig als kulturell Andere, kaum aber als politische Subjekte adressiert werden und damit strukturelle Benachteiligungen unsichtbar werden. Für einen geschärften Blick der rassismusund geschlechtertheoretischen Gesellschaftsanalyse halten wir es für vielversprechend, pädagogische Bemühungen um Sensibilisierung und Entnormierung von Geschlecht und Sexualität auch als Herrschaftstechnik zur Kenntnis zu nehmen, die in sehr unterschiedliche gesellschaftliche Kräftefelder integriert werden kann. In unserem vierten Punkt plädieren wir abschießend für eine Neuaneignung des raceBegriffs, um gegenwärtige Formen der Ethnisierung und Rassifizierung benennbar zu machen.

\section{LEITFRAGEN UND FORSCHUNGSDESIGN}

Zwei zentrale Fragestellungen leiteten unsere Fallstudie: Zum einen ging es uns um die gegenwärtige Ausgestaltung der gesellschaftlich-medialen Diskursproduktion, die Antifeminismus und Ethnisierung bzw. Rassismus miteinander verschränkt. 
Zum anderen interessierte uns, welche Effekte diese Diskurse auf die soziale Praxis von Orientierungskursen für neu Zugewanderte haben. Wir beziehen uns damit auf zwei unterschiedliche Datenquellen: Einerseits auf mediale Diskurse und andererseits auf spezielle Orte der politischen Bildung, die Teil staatlicher Integrationspolitik sind. Deshalb sind sowohl die Methoden der Datenerhebung als auch die Methoden der Auswertung von unterschiedlichen Herangehensweisen geprägt.

Für die Ermittlung der Diskurslandschaft auf medialer Ebene zogen wir theoretische und empirische Analysen aus der feministischen Theorie (Dietze 2016, 2017; Hark/Villa 2017; Rendtorff 2017; AK Fe.In 2019), aus der postkolonialen und rassismuskritischen (Migrations-)Forschung (Messerschmidt 2016; Mecheril 2017; Boulila/Carri 2017; Gutiérrez Rodríguez/Tuzcu/Winkel 2018) sowie Medien- oder Diskursanalysen heran (Keskinkılıç 2016a; Magni 2016; Dieterich 2016; DziubaKaiser/Rott 2016; Rams 2017; Fritzsche 2017). Angesichts der bereits vorliegenden (Diskurs-)Analysen entschieden wir uns für eine sekundäranalytische Re-Analyse der bereits vorliegenden Studien, die wir mit eigenem empirischen Material anreicherten. Die Auswahl des Textkorpus erfolgte nach dem Kriterium eines möglichst breiten Samples in Bezug auf Personen und Publikationsorgane aus der Parteienlandschaft und der Zivilgesellschaft. Als Grundlage dienten uns Parteiprogramme der AfD, Zeitungsartikel aus dem extrem rechten Spektrum, Beiträge aus sozialen Medien, die Kampagnen der Pegida-Bewegung und Schriften der Identitären Bewegung sowie Artikel aus Tageszeitungen und Wochenzeitschriften, die wir inhaltsanalytisch ausgewertet haben.

Auf dieser Basis identifizierten wir in der aktuellen Diskurslandschaft drei diskursive Verknüpfungen von Antifeminismus und Rassismus: Einen bevölkerungspolitischen Diskurs, in dessen Zentrum die Sorge um die national gedachte Familie steht; einen Diskurs, der die Überlegenheit der westlichen Geschlechterordnung rückständigen nicht-westlichen Geschlechtervorstellungen gegenüberstellt sowie einen Gefährdungs-/Verführungsdiskurs, der um den ,arabischen Anderen' als affektiv hoch besetzte Problemfigur kreist. Diese Diskurse lassen sich nur analytisch trennen und greifen empirisch nicht selten ineinander. Die gewonnene Einteilung soll die jeweiligen Anknüpfungspunkte analytisch verdeutlichen.

Ziel der Sichtung medialer Diskurse war es nicht, herauszufinden, $o b$ es eine diskursive Verbindung zwischen Antifeminismus und Rassismus gibt - dies arbeiten Gegenwartsdiagnosen bereits überzeugend heraus (vgl. Dietze 2016, 2017; AK Fe.In 2019). Es ging uns vielmehr darum, worauf diese Verschränkung verweist, was ihre argumentativen Bezugspunkte sind und wie sie in ihren unterschiedlichen Facetten beschrieben werden kann. Die Re-Analyse bietet damit die Möglichkeit herauszustellen, welche Stoßrichtungen die jeweiligen Diskurse haben, wie unterschiedliche Adressat_innen(-gruppen) davon angesprochen werden können/sollen und welche mobilisierenden Anschlussstellen sie bieten. 
Mit unserer zweiten Leitfrage wandten wir uns der Untersuchung von Orientierungskursen zu. Wir wollten dabei ermitteln, ob die medialen Diskurse auch auf der Ebene der sozialen Praxis des Kursgeschehens aufzufinden sind, und wenn ja, in welcher Form. Zudem ging es uns um subjektive Einschätzungen und Haltungen zur Debatte rund um Geschlecht, kulturelle Differenzen und zu ,Köln‘. Dabei wollten wir nicht zuletzt neu Zugewanderte, deren Positionen selten Ausgangspunkte für Gegenwartsanalysen sind, selbst zu Wort kommen lassen.

$\mathrm{Zu}$ diesem Zweck haben wir in mehreren deutschen Städten Orientierungskurse (d.h. den 100 Stunden umfassenden, letzten Teil von verpflichtenden Integrationskursen) teilnehmend beobachtet und zwar das dritte Modul Mensch und Gesellschaft. ${ }^{2}$ Wir haben mit Kursbeteiligten (Teilnehmenden, Programmverantwortlichen und Dozent_innen) Interviews geführt sowie Schulungsunterlagen und Lehrwerke ausgewertet. $^{3}$ Die Interviews konzipierten wir nach Witzel (2000) als problemzentrierte, leitfadengestützte Interviews mit einem auffordernden Charakter und erzählgenerierenden Fragen. Unsere Fragenkomplexe kreisten um Begriffe, die in der medialen Berichterstattung um die Ereignisse in Köln von zentraler Bedeutung waren: Geschlecht/Sexualität, Kultur, Werte und ,Köln` als Ereignis.

Wir haben Kurse in sieben Städten besucht und an jedem Standort eine unterschiedliche Anzahl an Interviews und Teilnehmenden Beobachtungen (TB) durchgeführt. Die Anzahl der Interviews mit Teilnehmenden (TN), mit Personen der Organisationsebene (OE) und mit Kursleiter_innen (KL) sind der unteren Tabelle zu entnehmen. Ziel war ein kontrastierendes Sample, das Klein- und Großstädte, Orte in Ost- und Westdeutschland, Kurse in unterschiedlicher Trägerschaft sowie eine ausgewogene Anzahl weiblicher und männlicher Interviewpartner_innen umfasst.

Die Auswertung der Interviews erfolgte inhaltsanalytisch (Mayring 2008) anhand der vier Kernthemen (Geschlecht/Sexualität, Kultur, Werte und ,Köln`). Durch die inhaltsanalytische Clusterung der Themen konnten wir zunächst auf einer expliziten und deskriptiven Ebene festhalten, wie die unterschiedlichen Kursbeteiligten auf die Themenkomplexe Bezug nahmen. Die teilnehmende Beobachtung hingegen erlaubte es uns, die soziale Praxis und Formen der Interaktion im Kurs miteinzubeziehen, in der manchmal auch implizite Annahmen deutlich wurden. Die Auswertung der Lehrmaterialien und Curricula betrachten wir hingegen als strukturgebendes Element, in dem Rahmungen und Zielvorstellungen der Kurse deutlich werden.

2 Der Integrationskurs dauert in der Regel 700 Unterrichtseinheiten, er besteht aus einem Sprachkurs (600 UE) und einem Orientierungskurs (100 UE). Der Orientierungskurs selbst besteht aus drei Teilen oder Modulen: Politik (Modul 1), Geschichte (Modul 2) und Mensch und Gesellschaft (Modul 3).

3 Eine Übersicht der in die Analyse einbezogenen Datenquellen findet sich im Anhang. 
Abbildung 1: Sample Orientierungskurse

\begin{tabular}{|c|c|}
\hline $\begin{array}{l}\text { Erhebungsdaten: } 33 \text { Interviews, } \\
11 \text { Teilnehmende Beobachtungen }\end{array}$ & 7 Städte \\
\hline 5 Interviews ( $1 \mathrm{KL}, 4 \mathrm{TN}) ; 1$ Kurs $-1 \mathrm{~TB}$ & Ort 1 Großstadt; VHS \\
\hline 6 Interviews (1 OE, $1 \mathrm{KL}, 4 \mathrm{TN}) ; 2$ Kurse $-2 \mathrm{~TB}$ & Ort 2 Großstadt; NGO \\
\hline 5 Interviews ( $1 \mathrm{OE}, 1 \mathrm{KL}, 3 \mathrm{TN}) ; 1$ Kurs $-1 \mathrm{~TB}$ & Ort 3 Großstadt; VHS \\
\hline 3 Interviews (1 KL, $3 \mathrm{TN}) ; 1$ Kurs $-2 \mathrm{~TB}$ & $\begin{array}{l}\text { Ort } 4 \text { kleinere Großstadt; } \\
\text { VHS }\end{array}$ \\
\hline $\begin{array}{l}6 \text { Interviews (1 OE, } 2 \mathrm{KL}, 2 \mathrm{TN}, 1 \text { Gruppegespräch mit } 8 \\
\text { Kursteilnehmer_innen); } 2 \text { Kurse }-2 \mathrm{~TB}\end{array}$ & Ort 5 Großstadt; VHS \\
\hline $\begin{array}{l}5 \text { Interviews ( } 1 \mathrm{OE}, 3 \mathrm{KL}, 1 \text { Gruppengespräch mit } 7 \text { Kurs- } \\
\text { teilnehmer_innen); } 2 \text { Kurse }-3 \mathrm{~TB}\end{array}$ & $\begin{array}{l}\text { Ort } 6 \text { mittlere Großstadt; } \\
\text { Basiskurs VHS }\end{array}$ \\
\hline 3 Interviews ( $1 \mathrm{KL}, 2 \mathrm{TN}) ; 1$ Kurs $-1 \mathrm{~TB}$ & Ort 7 Kleinstadt; VHS \\
\hline
\end{tabular}

Unsere Studie untersuchte den für das REVERSE-Projekt grundlegenden Analysebegriff des Antifeminismus somit auf seine intersektionale Verschränkung mit Rassismus und Migrationsfeindlichkeit hin. In Anlehnung an Gabriele Dietze (2017) legen wir den Schwerpunkt darauf, das argumentative Instrumentarium herauszuarbeiten, das die Abwehr und Abwertung feministischer und geschlechterpolitischer Forderungen mit rassistischen und migrationsfeindlichen Haltungen verbindet. Im antifeministischen Spektrum gibt es durchaus positive Bezugnahmen auf feministische Errungenschaften, die bisher Erreichtes (z.B. Frauenrechte) anerkennen, damit allerdings Geschlechtergerechtigkeit bereits als hergestellt ansehen und weitergehende Forderungen zurückweisen. Auf dieser Grundlage wird ein starker Gegensatz zu jenen ,kulturell Anderen“ konstruiert, die sich ihrer Ansicht nach noch nicht in dieser fortschrittlichen und egalitären Geschlechterordnung eingefunden haben.

Erst im Forschungsverlauf hinzugekommen ist der analytische Begriff Postfeminismus. Wir orientieren uns dabei an Zeitdiagnosen aus dem englischen Sprachraum (McRobbie 2010, 2011; Gill 2018), die in gegenwärtigen westlichen Gesellschaften die Entstehung eines neuen Geschlechtervertrags beobachten, der feministische und geschlechterpolitische Errungenschaften als bereits umgesetzt proklamiert. Die Möglichkeiten und Chancen, die sich einigen Frauen und einigen Angehörigen marginalisierter Gruppen damit eröffnen, basieren auf der stillschweigenden Übereinkunft, dass Widerstand gegen männliche Herrschaft nicht nur anachronistisch ist, sondern auch die eigenen Aufstiegschancen verstellt. Frauenpolitische Forderungen zum Beispiel bezüglich der Arbeitsmarktintegration von Frauen werden in spezifischer Form aufgegriffen und ins Scheinwerferlicht gerückt - und avancieren zum Zeichen von Modernisierung und Fortschritt. Ungelöste gesellschaftliche Probleme wie die Verteilung von Care-Arbeit oder geschlechtsbezogene Gewalt gegen Frauen werden geleugnet, ignoriert oder individualisiert. Für uns ist 
der Begriff des Postfeminismus mit Blick auf die soziale Praxis der Orientierungskurse interessant. Denn während offen antifeministische Diskurse oder Praktiken dort keinerlei Platz finden, lässt sich durchaus von einem postfeministischen Setting sprechen - hier stellt sich die Frage, ob dies nicht als „,sophisticated anti-feminism“ (McRobbie 2011: 179) bezeichnet werden muss oder zumindest einen äußerst fruchtbaren Boden dafür bereitet.

Werte, Wertorientierung, Wertevermittlung sind keine von uns in analytischer Absicht eingesetzten Begriffe, sondern stammen aus den Krisennarrativen rund um die Kölner Silvesternacht. Man könnte sogar sagen, dass sie zum Dreh- und Angelpunkt des Köln-Diskurses wurden, sei es im Zusammenhang mit der Sorge um einen drohenden Werteverlust, der Notwendigkeit einer klar formulierten ,Leitkultur ${ }^{6}$ oder in Bekenntnissen zu den demokratischen Grundwerten. Da verfassungsbezogene und alltagsrelevante Werte auch im Orientierungskurs eine wichtige Rolle spielen, haben wir die Kursleiter_innen und Programmverantwortlichen nach ihrem Verständnis des Werte-Begriffs, nach subjektiven Zugängen zum Thema gefragt sowie danach, ob sie sich als Wertevermittler_innen sehen. Deutlich wurde dabei eine sehr breite Palette an subjektiven Zugriffsweisen auf den Begriff ,Werte' sowie eine Vermischung dieses Begriffs mit ,Normen', ,Prinzipien', ,Konventionen“ oder ,Sitten ' - Vermengungen und Synonymsetzungen, die nicht nur die öffentliche, sondern auch die politische Wertedebatte der Gegenwart kennzeichnen, wie die aktuelle Bildungsforschung zeigt (Stojanov 2018: 64). Da dieser Begriff derart breit und unterschiedlich verwendet wird, eignet er sich unserer Ansicht nach nicht als analytischer Begriff, der helfen könnte, das Feld zu sortieren.

Ähnliches gilt für den Begriff der Kultur - ein Begriff, der in Wissenschaft, Politik und Öffentlichkeit generell höchst unterschiedlich verwendet und auch für ethnisierenden Zuschreibungen genutzt wird (Balibar 1992). In der Debatte rund um Köln fand er prominent Verwendung, indem kulturelle Differenzen zwischen der Mehrheitsbevölkerung und neu Zugewanderten entweder stark betont oder vehement zurückgewiesen wurden (Grubner 2016). Unter den Kursbeteiligten spiegelten sich die diversen in Umlauf befindlichen Kulturkonzepte: Von Kultur als Inbegriff für ,Werte und Normen einer Gesellschaft' über ein Verständnis, das stark an ,Tradition“ und ,Herkunft' gebunden ist bis hin zu Kultur als alltagsbezogenem Sinnsystem. Der Begriff der Kultur ließ sich - sowohl auf der diskursiven Ebene als auch im Feld der Orientierungskurse - nicht immer von Ethnizität und Nationalität abgrenzen bzw. wurde nicht klar von diesen Begriffen unterschieden. In der kritischen Migrationsforschung wurde dieses Phänomen bereits benannt, so verwendet beispielsweise Paul Mecheril (2002) die Begriffsbildung „natio-ethno-kulturell“. Zuschreibungen und Othering-Prozesse rekurrieren auf alle drei Ebenen und verweisen wechselseitig aufeinander. Kultur wird in diesem Zusammenhang nicht als fluide Alltagspraxis betrachtet, sondern es geht um ,Herkunft', um statische Eigenschaften von Menschen, was von Étienne Balibar als „Rassismus ohne Rassen“ 
(Balibar 1992: 28) beschrieben wurde. Der Begriff Kultur erfährt in diesem Zusammenhang eine rassistische Instrumentalisierung. Um diese Phänomene zu verdeutlichen, verwenden wir die Begriffe Ethnisierung und Rassifizierung. Beide bieten die Möglichkeit, den Prozess des othering in den Mittelpunkt der Analyse zu rücken (El-Tayeb 2016: 21).

Den Begriff ,Köln“ nutzen wir im Sinne von Gabriele Dietze (2016) als Kürzel für ein Ereignis, das letztlich auch diskursive Verschiebungen und Neuakzentuierungen hervorgebracht hat. Dietze hat als eine der ersten darauf hingewiesen, dass ,Köln“ in der gesellschaftspolitischen Debatte sehr schnell zu einer Chiffre avancierte, also zu etwas, das keiner weiteren Erklärung bedarf, damit ihre Bedeutung und ihre Signalwirkung verstanden wird. ,Köln“ scheint für eine spezifische Diskursverschiebung zu stehen, in der Ethnisierungsprozesse zugespitzt und inhaltlich verschoben werden: Es ist nicht mehr die ,muslimische Familie' oder die, islamisch geprägten Geschlechterverhältnisse', die als Kernproblem identifiziert werden, sondern vielmehr der ,muslimische Mann“ in seiner besonderen sexuellen Potenz und Gefährlichkeit.

\section{DiskURSIVE Formationen AM KREUZUngSPUNKT VON ANTIFEMINISMUS UND RASSIFIZIERUNG}

Im Folgenden skizzieren wir jene drei von uns aus der Sekundäranalyse der vorhandenen Forschungsliteratur und unserer ergänzenden Auswertung medialer Debatten herausgearbeiteten Diskurse, die Antifeminismus und Rassismus auf je unterschiedliche Weise verschränken.

\subsection{Der bevölkerungspolitische Diskurs}

Im Zentrum des bevölkerungspolitischen Diskurses steht ein Begriff der Familie, der zum Ausgangspunkt von Kritik und Sorge wird. Bestandteil dieser diskursiven Formation ist die Aufwertung von Müttern und der traditionellen Kleinfamilie; Feminismus und Geschlechterpolitik werden als anti-familial abgelehnt. Die als weiß imaginierte traditionelle deutsche Familie gilt dabei als „Keimzelle der Nation“ (Kemper 2014: 13). Es findet eine rassistische Entgegensetzung zu ,anderen' (v.a. muslimischen) Familien statt, deren „Geburtenstärke“ der „Reproduktionsverweigerung deutscher Frauen" entgegengesetzt und als Bedrohung inszeniert wird (Gutiérrez Rodríguez/Tuzcu/Winkel 2018). Die zunehmende Bedeutung der Familie als Angriffspunkt des neueren Antifeminismus wird in der wissenschaftlichen Literatur mit Begriffen wie „Familienfundamentalismus“ (Gesterkamp 2010), „Familienpopulismus“ (Lang 2015), (bürgerlich-konservativer) „Familismus“ (Notz 
2015) oder „familienzentrierter Antifeminismus“ (Scheele 2016) bezeichnet. Plakativ gesprochen richtet sich der familienfokussierte Antifeminismus einerseits nach ,innen“ (gegen Homosexualität bzw. Homo-Ehe, Adoption von Kindern durch nicht heterosexuelle Eltern, gegen queere Familien als Untergang traditioneller Werte, gegen pluralistische Sexualerziehung, gegen ,Frühsexualisierung' usw.), andererseits nach ,außen“ (Problematisierung und Skandalisierung des Bevölkerungswandels durch Migration, Globalisierung und Einwanderung). Die Familienzentrierung liefert dem Antifeminismus damit Anschlüsse an rechtsextreme und neonazistische Bedrohungsszenarien (Lang 2015: 171; Scheele 2016: 15). Die durch ,GenderIdeologie“ befeuerte „Paarungsmüdigkeit der Deutschen“ wird hier mit dem völkischen Motiv des ,Volkstodes', d.h. des Aussterbens des deutschen Volkes durch sinkende Geburtenraten, Abtreibung und Zuwanderung verbunden (Botsch/Kopke 2018: 64). Popularität gewann der bevölkerungspolitische Diskurs bereits durch die Publikationen von Eva Hermann (2006), Thilo Sarrazin (2010) und Akif Pirinçci (2014). Wie Ganz und Mesmer (2015: 72) hervorheben, eint diese - allesamt zu Bestsellern avancierten - Bücher eine biopolitische Logik, „die den Erhalt deutscher Werte an die Erhöhung der Geburtenrate in traditionellen, deutschen Familien koppelt."

Im Parteienspektrum zeigte lange Zeit nur die NPD eine offen völkischnationalistische Ausrichtung, die auf die Errichtung eines autoritären Nationalstaates auf der Grundlage einer ethnisch definierten Volksgemeinschaft zielt. Sie verknüpft ihre Familienvorstellungen unzweideutig mit Xenophobie, der Ablehnung der Ausweitung von Homosexuellen-Rechten (z.B. Adoption) und einer rassistischen Ideologie der Reinheit der Nation. In der AfD wurden offen rassistische Argumentationen zunächst vermieden, gemäß der ehemaligen Vorsitzenden Frauke Petry sah sich die Partei nicht als völkisch-rechte Partei. Ihr starker Fokus auf die Familie - und ihr Selbstverständnis als ,Familienpartei ${ }^{`}$ - richtet sich gegen die vorgeblich von der EU gesteuerte ,Gender-Ideologie“ oder ,Gender-Lobby', die insbesondere vom rechts-konservativen Flügel als Angriff auf die traditionelle heterosexuelle Mehrkindfamilie bekämpft wird (Lang 2015: 17f). Waren Migrationsabwehr und Rassismus in der AfD zunächst unterschiedlich akzentuiert - je nach neoliberaler, antisäkularer und neurechter Positionierung (Kemper 2014), lässt sich inzwischen von einer Durchsetzung neurechter Positionen in der Partei sprechen. Akteure wie Götz Kubitschek und Björn Höcke verbinden den Kampf gegen die ,Gender-Ideologie' klar mit rassistischen und völkischen Vorstellungen. Die AntiMigrations-Haltung der AfD verbindet sich in ihrem Wahlprogramm für die Bundestagswahl 2017 mit einer ,,aktivierenden Bevölkerungspolitik“, die familien- und bevölkerungspolitischen Maßnahmen für einheimische Familien ,,Vorrang vor Zuwanderung" einräumen möchte (AfD 2017: 29).

Deutlich zum Ausdruck kommt diese bevölkerungspolitische Sorge in der seit 2014 aktiven Bewegung Pegida (Patriotische Europäer gegen die Islamisierung 
des Abendlandes), die sich als Protestbewegung gegen die deutsche Einwanderungs- und Asylpolitik versteht. Eine Studie der Amadeu-Antonio-Stiftung hat öffentliche Äußerungen von Pegida aus geschlechterreflektierender Perspektive ausgewertet und zeigt die enge Verflechtung von Antifeminismus und Rassismus auf (Fachstelle Gender, GMF und Rechtsextremismus 2017).

Prominent findet sich das rassistisch-völkische Narrativ des ,Bevölkerungsaustausches' auch bei der rechtsextremen Identitären Bewegung unter dem Stichwort „der große Austausch“ (Identitäre Bewegung 2019; Erk 2019). Verwandte Äußerungen zur Sorge um die bevölkerungspolitische Entwicklung Deutschlands und Bedrohungs- bzw. Verschwörungsszenarien über einen bevorstehenden Bevölkerungsaustausch durch Einwanderung veröffentlichen auch andere rechtsextreme Zeitschriften und Blogs (Elsässer 2017; PI-News 2017 oder nationalistische Webseiten wie Zukunft Heimat [o.J.]).

Völkisch-rassistische Bevölkerungsvisionen sind zweifellos seit Langem Bestandteil des extrem rechten Spektrums. Allerdings ist nicht erst seit den jüngsten Zuwanderungen 2015 eine deutliche Ausbreitung solcher Denkfiguren zu bemerken, die weit in die Mitte der Gesellschaft hineinreichen. Es geht, wie Christoph Kopke bereits 2017 resümiert, nicht mehr um die „NPD und ihr Milieu“, sondern um eine inzwischen „breite und teils systemilloyale rechte Protestbewegung, die von bürgerlichen Akteuren bis tief in die extreme Rechte reicht." (Kopke 2017: 50) Diese kreist um die „ethnisch homogen imaginierte deutsche Nation“, die „durch innere und äußere Feinde“ (ebd.: 53) bedroht wäre. Neben der Dämonisierung von Eliten und der Gefahr der Islamisierung ist es insbesondere die Bedrohung durch eine angebliche Gender-Ideologie und das nahende Aussterben des deutschen Volkes, die zu den verbindenden Themen der nun sehr viel breiter aufgestellten rechtspopulistischen und rechtsextremen Bewegungen und Szenen zählen (Kopke 2017).

\subsection{Der geschlechterpolitische Überlegenheitsdiskurs}

Unter diesem Begriff versammeln wir Denkfiguren, die sich auf Feminismus und Geschlechterpolitik nicht (nur) als Verfallserscheinungen oder zerstörerische Kräfte (für Familie und Gesellschaft) beziehen. Vielmehr greifen sie feministische Errungenschaften (auch) als Inbegriff der Fortschrittlichkeit westlicher Gesellschaftsentwürfe auf, um einen Kontrast zu nicht-westlichen Anderen (als archaisch, rückständig, unterlegen) herzustellen. Durch den Hinweis auf die angeblich auf der Gleichstellung von Frauen und Homosexuellen beruhenden westlichen Geschlechterordnung wird ein Überlegenheitsnarrativ konstruiert, das zur Abgrenzung und Abwertung von nicht-westlichen Geschlechtervorstellungen genutzt wird.

Wir haben es hier mit einem Diskurs zu tun, der sich auf den ersten Blick kaum als antifeministisch lesen lässt - verweist er doch mit Stolz auf die Leistungen der 
Frauen- und Schwulenbewegung. In die wissenschaftliche Debatte wurde diese diskursive Konstruktion - zumindest Teile davon - zunächst unter den Begriffen Homonationalismus (Puar 2007) und Femonationalismus (Farris 2011) eingeführt. Beide Begriffe zielen auf die Kritik der Instrumentalisierung emanzipatorischer Bewegungen $a b$, wenn westliche Nationen die Unterwerfung anderer Länder im Namen der Befreiung von Homosexuellen oder Frauen forcieren. Ein Kennzeichen dieser Indienstnahmen ist die Verbindung eines kulturalistisch argumentierenden Rassismus mit postfeministischen Zügen: Die Freiheit von Frauen und Homosexuellen, so die Botschaft, ist in den westlichen Gesellschaften bereits erreicht. Eine emanzipatorische Veränderung der Gesellschaft ist aus dieser Sicht nicht notwendig, vielmehr gilt es, das Erreichte nun gegen Bedrohungen von außen zu verteidigen.

Dabei kann es durchaus vorkommen, dass ein und dieselben Akteur_innengruppen beide Bezüge auf den Feminismus - Feminismus als Verfallserscheinung und Feminismus als nationales Markenzeichen - vertreten, wie Gabriele Dietze (2017) anhand des Pegida-Manifests aus dem Jahr 2014 zeigt: Es spricht sich gegen die ,wahnwitzige Genderisierung‘, gegen ,Gender-Gaga‘ und ,Genderismus ‘ aus und zugleich ,gegen frauenfeindliche Ideologien' und, für sexuelle Selbstbestimmung‘. Damit gelingt es, so Dietze, zwei strategische Ziele miteinander zu kombinieren: die Zurückweisung von Forderungen zum Abbau bestehender geschlechterhierarchischer Strukturen und die Zurückweisung von Einwanderung, die als Gefährdung der bestehenden Geschlechtergleichheit präsentiert wird. Dietze prägt diesbezüglich den Begriff ,,abendländischer sexueller Exzeptionalismus“ (2017: 207), der in solchen Diskursen gegen Geflüchtete und Zuwandernde in Stellung gebracht wird, insbesondere gegen Muslim_innen (Dietze 2017: 99f; vgl. zu verwandten Strategien aus anderen Kontexten Ahmed 1992: 144f und Scott 2007: 54f). Kommentare, die eine dichotome Entgegensetzung zwischen einem gleichberechtigten, aufgeklärt-westlichen Geschlechterverhältnis und einer islamischen Kultur sehen, die sich durch einen rückständigen, potenziell gewaltvollen Blick auf Frauen auszeichne, finden sich quer durch alle Parteien und in zahlreichen journalistischen und Debattenbeiträgen (AfD 2017; Palmer 2015; Buschkowsky 2015; focus 2018; rp online 2016; Krüger 2016; Emma 2015). Wir beschränken uns hier auf solche Beiträge, die diese Dichotomie mit der Ablehnung und Diffamierung über gegenwärtige Forderungen oder Kampagnen aus Feminismus und Geschlechterpolitik verbinden.

Exemplarisch kommt die Verknüpfung der Ablehnung der ,Gender-Ideologie“ mit der Kritik an ,rückständigen Geschlechtervorstellungen' rassifizierter Anderer unter dem Titel Mal ernsthaft: Mit Gendergaga gegen das arabische Frauenbild? zum Ausdruck. Angekündigt wurde damit eine Veranstaltung, zu der zwei CDUAbgeordnete im März 2016 geladen hatten (Lämmel/Vaatz 2016). Ein ähnliches Argumentationsmuster, das die Zurückweisung der ,Gender-Ideologie‘, mit ,Über- 
treibungen“ wie \#metoo, \#ausnahmslos oder der Ablehnung der Beschäftigung mit ,überflüssigen` Themen verbinden, finden sich in den Reihen der AfD (Presseportal der AfD 2016; Poggenburg 2018). In dem Vortrag einer AfD-Abgeordneten weist das geschlechterpolitische Überlegenheitsnarrativ auch eine Kehrseite auf: Durch feministische Errungenschaften sei (weißen, europäischen) Männern ihre Wehrhaftigkeit abhanden gekommen (Bilge 2018). Nach der Kölner Silvesternacht wird dieses Narrativ und die Forderung nach Remaskulinisierung häufig bemüht (vgl. dazu auch Rendtorff 2017).

Auch in der CDU wird die Gefahr von Frauenfeindlichkeit, Homophobie oder Antisemitismus durch muslimische Einwanderer mit den als überzogen markierten Forderungen von Feminismus und Geschlechterpolitik kontrastiert. „Es kann gar nicht genug gegendert werden, der Feminismus siegt sich bis in die Vorstandsetagen politisch korrekt zu Tode, aber bei Zwangsheirat, Burka und Ausgehverbot werden Frauenrechte dann doch relativ, man müsse Rücksicht nehmen auf die andere Kultur", lässt sich Jens Spahn bereits 2014 zitieren (Spahn 2014). Auch Christina Schröder (2016) zeigt sich, angesichts der in muslimischen Ländern „stark geprägte[n] Vorstellung von männlicher Ehre“ und der „höhere[n] Neigung zu Gewalt“, bestürzt über die Naivität von \#ausnahmslos-Unterstützerinnen (Schröder 2016).

An einigen dieser Zitate ist zu erkennen, dass das Überlegenheitsnarrativ keine Neuerfindung der letzten Jahre ist. Allerdings hat es seit den Ereignissen in der Kölner Silvesternacht 2015/16 eine starke Popularisierung erfahren.

\subsection{Der Gefährdungs-/Verführungsdiskurs}

Mit dem Blick auf einen Diskurs der Gefährdung und Verführung möchten wir eine Dimension hervorheben, die im öffentlichen Diskurs um ,Köln` besonders deutlich hervortritt. Im Nachgang wurde zwar die Gefährdung durch den (verallgemeinerten) arabischen Anderen in den Fokus gerückt, dennoch lässt sich anhand der medialen Aufbereitung auch der Topos der Verführung nachvollziehen. Im Brennpunkt dieses Diskurses steht die rassifizierte Figur des ,fremden Mannes', die heute insbesondere um den jungen muslimischen Mann kreist, jedoch Anleihen bei sehr viel älteren Imaginationen des ,Schwarzen Mannes` nimmt, wie sie beispielsweise von Frantz Fanon (2008) beschrieben wurden.

Mit dem Gefährdungs-/Verführungsdiskurs fokussieren wir ein historisch wiederkehrendes Muster, wonach Männer(-gruppen) rassistisch herabgesetzt werden sollen, indem ihnen eine besondere sexuelle Potenz und Gefährlichkeit zugeschrieben wird (Rendtorff 2017: 302). Es ist ein markantes Element kolonialer Sexualpolitik und nationalistischer Propaganda (vgl. dazu von Braun/Mathes 2007) und spielt auch in der gegenwärtigen medialen und gesellschaftspolitischen Auseinandersetzung um muslimische Einwanderer eine deutliche Rolle (Rendtorff 2017: 
303; Hark/Villa 2017: 46f; Dietze 2016: 95f). In zahlreichen Debattenbeiträgen wurde die Figur des islamischen jungen Mannes mit sexuellem Exzess und sexueller Bedrohung verknüpft. In einem zuerst in La Republica erschienen Text, der für die FAZ ins Deutsche übersetzt wurde, attestiert der Journalist Kamel Daoud der arabisch-muslimischen Welt ein „krankes Verhältnis zur Frau, zum Körper und zum Begehren“ (Daoud 2016a), in der Zeit eine ,unterdrückte, aggressive Sexualität“" (Daoud 2016b). Die Autorin und Filmschaffende Güner Balci antwortet auf die Frage, wie man „eine Veränderung im Kopf des muslimischen Mannes“ erreiche:

„Es geht immer nur um Sex. Diese Obsession mit dem Sex ist eine, die extrem verbreitet ist in patriarchalischen Gesellschaften. Und es dreht sich nur um die Kontrolle der Frau und die Sanktionierung von Sex und darum, wie man ihn dennoch heimlich ausleben kann. Ich meine, die jungen Männer gehen in den Dschihad, um Sexsklavinnen zu haben, das ist für viele einer der größten Anreize.“ (Balci 2016: o.S.)

Für den Autor Samuel Schirmbeck gehört ,die sexuelle Gewalt in Nordafrika und im Nahen Osten zum Alltag“. Für ihn sind ,sexuelle Übergriffe [...] in islamischen Ländern die Regel und nicht Ausnahmen.“ (Schirmbeck 2016) Die feministische Publizistin Alice Schwarzer bringt die drohende Gefahr unter der Headline $\mathrm{Ka}$ laschnikows, Sprenggürtel und jetzt die sexuelle Gewalt (Schwarzer 2016) auf den Punkt und Ex-Femen-Aktivistin Zana Ramadani rügt muslimische Mütter, die nicht zuletzt das sexuell übergriffige Verhalten ihrer Söhne durch ihre Erziehung und Wertevermittlung zu verantworten hätten (Ramadani 2016). In einem FacebookEintrag des Thüringer AfD-Sprechers Björn Höcke vom 05.01.2016 heißt es: „Die Silvesternacht hat unserem Land mit den Ereignissen am Kölner Hauptbahnhof einen Vorgeschmack auf den drohenden Kultur- und Zivilisationszerfall gegeben. Hunderte Frauen wurden Opfer einer Gruppe von 1000 (!) nordafrikanischen, jungen Männern.“ (Höcke 2016)

Die Betonung der sexuellen Gefahr zog einen rassifizierenden Schutzdiskurs nach sich. Dietze (2016: 98) spricht von einem „Belästigungs-AusweisungsKomplex“, in dem sich autochthone, heterosexuelle, weiße Männer als „Spezialisten für ,Schutz“" etablieren (vgl. auch Völker 2016 und Clemm/Hark 2016).

Dieser Diskurs betont nicht nur Gewalt und Bedrohung, sondern es schwingt meist auch der Topos der Verführung mit: Der sexualisierte muslimische Mann ist nicht einfach ein ,roher Gewalttäter', sondern auch durch übermäßige sexuelle Potenz oder starke Verführungskraft gekennzeichnet (Fanon 2008; Gutiérrez Rodríguez 2016; Scott 2007). Solche Assoziationen schwingen deutlich in rechtskonservativen Appellen mit. „Mädls wacht auf! Der ,edle Wilde“ ist eine Illusion“, warnt etwa ein Autor in der österreichischen Zeitschrift Wochenblick (Rabeder 2019). 
Aufschlussreich sind diesbezüglich aber auch schulische Interventionsprojekte, die darauf abzielen, deutsche Mädchen davor zu bewahren, auf die Attraktivität von Einwanderern hereinzufallen. Ozan Keskinkılıç (2016a: 65) zitiert die Fachgewerkschaft der Gymnasiallehrer in Sachsen-Anhalt, für die die Anwesenheit von, wie sie formulieren, ,jungen, kräftigen, meist muslimischen Männern“ zu folgender Problematik führt: „Wie können wir unsere jungen Mädchen im Alter ab 12 Jahren so aufklären, dass sie sich nicht auf ein oberflächliches sexuelles Abenteuer mit sicher oft attraktiven muslimischen Männern einlassen?“. Andernorts ergeht von Schulleitern via Rundschreiben die Bitte an Schülerinnen, keine „durchsichtigen Tops oder Blusen, kurze Shorts oder Miniröcke“ anzuziehen, da dies zu Missverständnissen mit den in der Schulturnhalle untergebrachten Geflüchteten führen könne (Greiner 2015).

In der Zusammenschau lässt sich sagen, dass jeder dieser drei Diskurse ein anderes Motiv akzentuiert - die national gedachte Familie, die Fortschrittlichkeit des deutschen bzw. europäischen Geschlechterregimes, sexuelle Gefahr und Potenz des ,fremden Mannes ${ }^{\text {' }}$ - dass sie jedoch auch miteinander verknüpft auftreten. ${ }^{4}$ Im folgenden Abschnitt wenden wir uns der sozialen Praxis von Integrationskursen zu und fragen danach, ob sich Effekte solcher Diskurse auf der Ebene staatlicher Orientierungskurse finden.

\section{Sichtweisen Und Soziale Praxen IN ORIENTIERUNGSKURSEN}

Grundsätzlich sind die Orientierungskurse, die Teil der staatlich verpflichtenden Integrationskurse für neu Zuwandernde sind, der Vermittlung demokratischer, verfassungsbezogener und staatsbürgerlicher Rahmenbedingungen des Lebens in Deutschland gewidmet. Antifeministische oder rassistische Haltungen, Weltanschauungen oder politische Ideologien sind in unserem Sample nicht aufgetreten. Sie werden als solche auch von Seiten der beteiligten Organisationen abgelehnt. Was sich in der sozialen Praxis der Kurse aber zeigt, sind Spannungsverhältnisse, die aus einzelnen Denkfiguren der öffentlichen Debatte resultieren. Bisweilen treten Fragmente der oben analysierten Diskurse recht unmittelbar zu Tage (4.1). In anderen Fällen wird deutlich, dass die Übung in gesellschaftlicher Teilhabe strukturelle Problemlagen weitgehend ausspart (4.2). Es zeigt sich aber auch, dass einige Kursleiter_innen um die Benennung von strukturellen Benachteiligungen bemüht sind -

4 Ein Beispiel für die Verquickung aller drei diskursiven Motive findet sich auf der rechtsextremen Seite Die Sezession (Sezession 2015). 
was insbesondere ihrem persönlichen Engagement und weniger der Ausrichtung von Curricula und Lehrwerken geschuldet ist (4.3).

\subsection{Widerspiegelungen ethno-sexistischer Diskurse}

Während sich der Fokus in der öffentlichen Migrations- bzw. Integrationsdebatte von der Problematisierung der Geschlechterverhältnisse in migrantischen Familien nach Köln deutlich auf die sorgebereitende Figur des ,jungen muslimischen Mannes' verschoben hat, finden wir in der sozialen Praxis beide Themen als problematische Kernfragen vor. Dies wird durch Akzentsetzungen im Curriculum, in zwischenmenschlichen Interaktionen, in der konkreten Strukturierung des Unterrichts, in manchen Lehrwerken und in Aussagen von Beteiligten deutlich.

Ort 3 ist eine Stadt mit hoher GIDA-Präsenz. Dies zeigt sich in unserer Fallstudie nicht im Kursgeschehen selbst und weniger in den Interviews mit den Kursverantwortlichen, sehr deutlich aber in den Interviews mit den Teilnehmenden. Nach Ansicht der Programmverantwortlichen (Ort3/OE) gehe es im Orientierungskurs weniger um Sprachvermittlung, Ziel sei vielmehr die Vertiefung der Vermittlung von Regeln, Werten, Pflichten, Rechten. Sie führt die Erweiterung des Kursumfangs von 60 auf 100 Stunden auf die größeren kulturellen Differenzen zwischen der jetzigen Einwanderungsgruppe und der deutschen Mehrheitsgesellschaft zurück. Geschlechterrollen wären dabei der Knackpunkt, was sich auch im Kursgeschehen oder bei Anmeldung und Beratung zeige. Der am gleichen Ort interviewte Kursleiter (Ort3/KL) sieht den Grund für die Aufstockung darin, dass den Menschen mehr Zeit für ein gelingendes Ankommen gegeben werden sollte. Insbesondere die nun eingeräumte Zeit für Exkursionen bewertet er sehr positiv. Neben dem politischen Teil ist für ihn das dritte Modul, die Thematisierung von Gesellschaft und Kultur, „das Schöne [...], die Krönung von dem Orientierungskurs“ (Ort3/KL: 3). Hier gehe es um verschiedene Kulturen, Traditionsbilder und Familienbilder, die fest im Kopf verankert seien. Sein Motto dabei sei es nicht, die Meinung der TN zu verändern, aber Empathiefähigkeit zu erzeugen. Er hebt seinen Schwerpunkt auf Meinungsfreiheit und Rechte hervor und möchte dazu motivieren, über bestimmte Dinge nachzudenken, z.B. hinter eine „Religionswand“ zu blicken (ebd.). Wichtige Schwerpunkte seien außerdem Ausbildung, persönliche Freiheit, Frauen- und Männerrollen (Ort3/KL: 10).

Für OE und KL in Ort 3 ist die Thematisierung von Geschlechterthemen im Kurs wichtig - jedoch aus unterschiedlichen Gründen: Für die OE stellt das Geschlechterwissen den Kern dafür dar, wie man im neuen Land anderen Menschen begegnet, welche Menschen es hier gibt, was toleriert wird, was man kann, darf oder muss und auf welchen Gesetzesgrundlagen dies beruht. Dieses Wissen ist „wichtig, um überhaupt miteinander [...] leben zu können“ und wenn wir nicht 
möchten, dass „Parallelgesellschaften entstehen, wo man nach seinen Werten lebt und wo die anderen Werte keine Rolle spielen.“ (Ort3/OE: 26) Dass Geschlecht ein Knackpunkt für misslingende Kommunikation mit den neu Zuwandernden ist, illustriert die OE anhand von Schwierigkeiten am Kursort: Es käme zu Konflikten, weil Männer ihren Frauen die Kursanmeldung verweigerten oder bestimmen wollten, in welchen Kurs (idealerweise mit männlichen Verwandten) die Frauen kämen. Weitere Probleme ergäben sich daraus, dass männliche TN weibliche KL nicht respektierten und manchmal aus Beleidigungen weiblicher durch männliche TN (Ort3/OE: 9). Die Thematisierung von Geschlechterfragen im Kurs hätte daher das Ziel, gegenseitiges Verständnis hervorzubringen. Es ginge nicht darum, zu erwarten, dass das, was wir für gut und richtig halten, gänzlich übernommen würde, sondern um die Vermittlung dessen, was in Deutschland Gang und Gäbe ist, um die Minimierung von Missverständnissen und um das Finden eines Mittelweges (Ort3/OE: 25).

Die Programmverantwortliche sieht die Herausforderung durch eine kulturelle Differenz begründet, die sich in den Geschlechterverhältnissen zeige. Den Kulturbegriff bestimmt sie dabei folgendermaßen: „Der Begriff Kultur ist ja wirklich ein weiter Begriff. Ich würde da Werte drunter ordnen, Verhaltensweisen, Ansichten, die, die einen Menschen in einer bestimmten, aus einer bestimmten Herkunft, sag ich um nicht das Wort Kultur zu benutzen, geprägt haben“ (Ort3/OE: 16). Je größer die kulturellen Unterschiede (zwischen der einwandernden Gruppe und den Deutschen), desto größer sei auch der Bedarf nach Orientierung. Eine Rolle spiele allerdings auch das Stadt-Land-Gefälle: Je weiter weg jemand von der Stadtgesellschaft gelebt habe, desto stärkeren Halt brauche er/sie. Problematisch in Bezug auf kulturelle Unterschiede seien nicht nur Geschlechterkonflikte, sondern auch der Umgang mit Kritik (die z.B. mit einem Rassismus-Vorwurf zurückgewiesen werde) und darüber hinaus, dass Krieg und andere Probleme der TN aus dem Kursgeschehen ausgespart bleiben müssten (Ort3/OE: 12).

Der KL äußert sich dagegen skeptisch zur öffentlichen Debatte über den Kulturbegriff und lehnt die damit oftmals verbundene Erwartung ab, Migrant_innen sollten sich anpassen. In seiner Kurspraxis legt er den Akzent auf die Alltagskultur und die Individualität des/der Einzelnen, die er im Kurs als „Mensch aus Knochen, Blut und Haut“ (Ort3/KL: 8) anzusprechen sucht. Dieser Individualität stehen tradierte Familienbilder und religiöse Verhaftungen gegenüber. „Also Kulturbegriff so in dem Sinne. [...] Ich lebe meine persönliche Freiheit. [...] Dieses nichtkollektivistische, sondern eher ja, wie heißt das, individualistische. Das ist auch schon so ein eigentlich großer Unterschied zu vielen Personen, die hier her kommen." (Ort3/KL: 10) Im Kurs versucht er zu vermitteln, dass keine Seite das Recht hat zu erklären, wie es besser funktioniert. Kultur sei eigentlich alles, was uns umgibt (Ort3/KL: 9). Der Unterschied zwischen diesen beiden Kulturverständnissen liegt darin, dass die OE einen normativen, statischen Kulturbegriff verwendet, der Ausdruck in den Geschlechterverhältnissen findet. Dagegen bietet der Kurslei- 
ter einen individualistisch-freiheitlichen Zugang, der Kultur auch als Alltagspraxis benennt.

In Ort 3 zeigt sich eine deutliche Kluft zwischen den Interviews mit OE, KL und den Teilnehmenden. Nicht weil die TN den Kurs schlecht bewerten würden im Gegenteil, bis auf den Wunsch nach mehr praktischem Alltagswissen sind ihre Reaktionen sehr positiv. Die TN kommen jedoch teilweise direkt zu Beginn des Interviews auf massive Rassismuserfahrungen im Alltag außerhalb des Kurses zu sprechen. TN2 berichtet von ihrem ersten halben Jahr in Deutschland, als sie noch Kopftuch trug. Mehrmals sei sie auf der Straße beschimpft und angespuckt worden, sodass sie beschlossen hat, das Kopftuch abzulegen. Sie habe dies mit ihrem Vater diskutiert, der ihr zustimmte. Seither komme es zwar manchmal vor, dass jemand böse schaue, aber es sei nie mehr so schlimm wie früher geworden (Ort3/TN2: 21). Offensichtlich, so sagt sie, ist es in Ort 3 besonders schlimm, denn sie hat Cousinen in anderen Großstädten, die keine solchen Erfahrungen haben und fast nicht glauben könnten, wie es hier sei. Auch TN3 berichtet davon, angespuckt worden zu sein, eine Erfahrung, die er als besonders bitter beschreibt, da seine beiden kleinen Kinder anwesend waren. Dass Menschen die Straße wechseln, Beschimpfungen ausstoßen oder ihm in der Straßenbahn aus dem Weg gehen, wird zur alltäglichen Erfahrung. Am Ende des Interviews sagt er: „Die Leute wollen mich nicht. Die Leute brauchen sie mich nicht.“ (Ort3/TN3: 17)

Auch die OE berichtet davon, dass die TN sich bisweilen aufgrund der Proteste nicht mehr in den Kurs trauten. Ihre Ängste werden sowohl von OE als auch vom $\mathrm{KL}$ als berechtigt betrachtet, allerdings nur für Erfahrungen außerhalb des Kurses. Es gebe bisweilen Rassismusvorwürfe der TN gegenüber dem Team des Kursstandortes, die sich aber, so fügt sie hinzu, im Vieraugengespräch nicht selten als Missverständnis herausstellen würden (Ort3/OE: 29, 30).

Auch in Ort 4 gilt die Thematisierung der Geschlechterverhältnisse als zentrales Merkmal für das Gelingen von Integration. Für das Modul Mensch und Gesellschaft greift der KL nur punktuell auf die Lehrwerke zurück. Teilweise empfindet er sie als „,von oben herab“ und „,zermürbend“ (Ort4/KL: 22; vgl. auch Ort4/TB1: 1), weil immer wieder die gleichen Fragen gestellt würden, die von einem klischeebehafteten Bild von Migrant_innen ausgingen. Als Beispiel nennt er das beständige Wiederholen des Themas Pünktlichkeit. Die Fragen im Buch würden darauf abzielen eine Antwort zu geben die hervorhebt, wie wichtig Pünktlichkeit ist. Diese Fragen würden von den Teilnehmer_innen auch immer richtig beantwortet. Trotzdem kämen sie dann zum Kurs immer zu spät (Ort4/TB1: 1). Er schlägt deswegen vor, dass die Anwesenheitspflicht durch Sanktionen gestärkt werden solle. Es geht ihm weniger um die klischeehafte Darstellung, als mehr darum, dass er es als notwendig empfindet, mit Direktiven zu arbeiten. Nur so könne man tatsächlich Probleme in der Migrationsgesellschaft ansprechen. Er bringt daher eigene Beispiele ein, um, wie er hervorhebt, die wahren Konfliktlinien zu zeigen, die spezifische Mig- 
rant_innengruppen besonders beträfen. Die Lehrbücher würden keine Diskussion anregen und seien deshalb für diese Fragen weniger geeignet. Dass „,wir“ uns in der Thematisierung von Sexualität und Geschlecht ein wenig auf dem „hohen Ross“ bewegen würden, hebt er zwar hervor, dennoch ist die fortschrittliche Toleranz im heutigen Deutschland für ihn eine unbestreitbare Tatsache (Ort4/KL: 13).

„Ja. Zum Beispiel unsere Akzeptanz von Homosexualität. Ja, da. Oder, oder unsere Akzeptanz von Frauenrechten. Noch in den 50er Jahren war es so, dass auch in Deutschland die Frau nur arbeiten durfte, wenn der Mann zugestimmt hat. Jetzt sind wir immer so ein bisschen auf dem hohen Ross gegenüber anderen Kulturen, wenn die das nicht machen. Aber wir sind auch noch nicht ewig, so wie wir heute tolerant sind.“(Ort4/KL: 13 )

Sein Bemühen ist also von der Haltung geprägt, echte Konfliktlinien beim Thema Geschlechterungleichheit zu zeigen; ein Überlegenheitsnarrativ ist ihm durchaus bewusst, dennoch sieht er ,uns' nun als tolerant an. Im Unterricht arbeitet er mit zwei Texten, die auf das Thema Ehe und Familie eingehen. Zunächst geht es um die Frage, wer wen heiraten darf. In der Diskussion zur gleichgeschlechtlichen Ehe geht es im Kurs hoch her. Einige erzählen, dass sie aus Ländern kommen in denen dies verboten ist. Die meisten sprechen sich für die gleichgeschlechtliche Ehe aus und erzählen von eigenen Erfahrungen mit dem Thema, nur zwei Frauen argumentieren dagegen. Eine hält Homosexualität für ein hormonelles Problem, das sich leicht beheben ließe. Der anderen Teilnehmerin fällt es schwer sich auf Deutsch zu artikulieren; sie macht ihrem Missfallen mit emotionalen Ausrufen Luft. Der KL ist mit dem emotionalen Ausbruch dieser Frau überfordert. Nachdem die anderen TN sie mehrfach darauf hingewiesen haben, dass es hier in Deutschland nicht richtig sei, so zu sprechen, tritt er an ihren Tisch und hält eine recht emotionale Rede. Danach entlässt er den Kurs in die Pause.

Am zweiten Tag arbeitet der KL mit einem Zeitungsartikel zum Thema Ehrenmord. Die TN sind schockiert, dass es so etwas in einem ,aufgeklärten' Land wie Deutschland gibt. Die Fragen konzentrieren sich auf die Umstände der Familie und auf die religiös-kulturelle Herkunft des getöteten Mädchens. Das Mädchen, dessen Familie mutmaßlich muslimischen Glaubens ist, kann ihre Sexualität nicht frei ausleben. Er hebt hervor, dass dies ein zentraler Konflikt einiger Migrant_innengruppen sei und Deutsche wesentlich freier in der Auslebung ihrer Sexualität sind. Er fragt in die Runde ob die anderen Teilnehmer_innen das ähnlich sehen und wird darin bestätigt, dass Deutsche wesentlich freizügiger sind. Als Bild nehmen die TN die besondere Freizügigkeit der Deutschen und die Gewalt der muslimischen Anderen mit.

Dass der ,Köln-Diskurs' rund um die Gefährdung und Verführung durch den arabischen Anderen auch auf die zwischenmenschliche Interaktion im Kurs wirken kann, zeigen die folgenden Beispiele. 
Der KL in Ort 6 ist ein erfahrener und engagierter Lehrer, der selbst vor dreißig Jahren nach Deutschland migriert ist. In den 1990er Jahren hat er die rassistischen Pogrome in Rostock und Mölln und die Veränderung des gesellschaftlichen Klimas miterlebt. Das Thema Frauen, so sagt er, habe er im Kurs tausendmal zum Thema gemacht (Ort6/KL 2: 23). Angesichts der seit Köln erneut veränderten gesellschaftlichen Stimmung sehe er es als seine Pflicht an, mit den Teilnehmer_innen darüber zu sprechen. Der Hintergrund ist die Befürchtung, dass die gesellschaftliche Stimmung erneut kippt und so erklärt er, wie er Pressemitteilungen zur Kenntnis nehme:

Bei Berichten über sexualisierte Gewalt fragt er sich etwa: „Na, war es ein Syrer? War es ein Nigerianer? War es das? War es das?“ (Ort6/KL 2: 25) Berichte über Gewalttaten, die an Köln erinnern, haben für ihn eine soziale Sprengkraft, die ihn sichtlich in Angst versetzt. Als ihm ein TN erzählt, bei einem seiner Kneipenbesuche habe er die Erfahrung gemacht, dass Frauen ostentativ aufgestanden und weggegangen seien, als er an den Tisch kam, sagt er ihm Folgendes:

„Siehst du, du fühlst dich jetzt verletzt. Ich verstehe das. Ist auch nicht angenehm. Mit Sicherheit nicht. Aber, wenn ich dann so sehe, was so überall passiert, was glaubst du was diese Frauen wohl gerade gedacht haben? Sie kennen dich ja gar nicht. [...] [Es gibt] da gewisse Ängste oder so. [...] Viele deutsche Frauen oder europäische Frauen, polnische, russische, tschechische, französische haben Beziehungen mit Leuten aus dem Orient. Ist nicht so, dass sie es nicht haben. [...] Nur, wenn solche Beispiele [wie Köln; Anm. DBC/BG] kommen dann ist es schlecht. Und dann entsteht ja genau das. Sie stehen auf, weil du dich da hingesetzt hast." (Ort6/KL2: 25)

Der TN erhält also die Erklärung, dass er zur Gruppe derer gehöre, die momentan im Diskurs als gefährlich markiert werden. Mit anderen Worten ausgedrückt: Der KL zieht, obwohl er es nicht will und eigentlich darüber aufklären möchte, eine ethnisierende Unterscheidung, deren Zurückweisung kaum möglich erscheint.

Dass der Diskurs um Köln diese Wirkung der ,Gruppenhaftung‘ besitzt, kann auch die folgende kurze Episode erhellen, die uns die Kursleiterin von Ort 2 erzählt. In einem berufsbegleitenden Kurs hätten die Teilnehmenden die Vorfälle von sich aus angesprochen und deutlich gemacht, dass diese nichts mit der arabischen Kultur zu tun hätten. „Ich habe diese Geste so verstanden,“ erläutert sie, ,als würden sie mich beruhigen wollen, dass das nicht Standard ist und nicht. Und dass sie das selber schlecht finden und, und verurteilen. Und, ähm, es war nicht wirklich ein Thema hier." (Ort3/KL1: 15)

Auch in einem Gruppengespräch mit Kursteilnehmer_innen aus Ort 5 wurde deutlich, dass besonders die männlichen $\mathrm{TN}$ - die dem , arabischen Typ“äußerlich am nächsten kamen - unter der veränderten Atmosphäre leiden. Auf die Frage, ob sie sich in Deutschland willkommen gefühlt haben, antwortet ein männlicher TN: „Ja, alle waren nett bis 2015 in Ort 5. Nach 2015 nicht so gut für die Ausländer.“ 
(Ort5/TN-Gruppe: 6) Ein weiterer männlicher Teilnehmer ergänzt: „Weil Silvester." (ebd.) Die anderen nicken zustimmend, während zwei Teilnehmerinnen betonen, dass die Deutschen zu ihnen weiterhin ,nett“ seien (Ort5/TN-Gruppe: 7). Für eine andere Teilnehmerin aus der Gruppe hat die Nettigkeit allerdings etwas damit zu tun ob man gut deutsch spreche; sie hofft, dass sich ihr Deutsch bald verbessert.

\subsection{Der Orientierungskurs als Demokratie-Lernen}

Deutlicher Schwerpunkt in den Kursen - sowohl im direkten Kursgeschehen, als auch in den Interviews mit Beteiligten und den Lehrwerken - ist es, gesellschaftliche Teilhabe zu adressieren. Der Schwerpunkt liegt auf einem liberalen WerteDiskurs, der Meinungsvielfalt, Freiheit und Toleranz in den Vordergrund rückt.

Dies möchten wir am Beispiel der KL aus Ort 1 verdeutlichen. Für die KL ist das Geschichte-Modul weitaus bedeutsamer als das Modul Mensch und Gesellschaft, um etwas über Deutschland zu lernen. Sie zeigt sich in Bezug auf die öffentliche Kultur- und Wertedebatte skeptisch. Sollte es Werte geben, die sie vermitteln möchte, so seien dies Respekt und Toleranz. Es ginge darum, über Dinge zu sprechen, ohne gleich zu beurteilen, ob sie richtig oder falsch seien (Ort1/KL: 13). Sogar die in anderen Kursen als ,traditionell' und ,überkommen' diskutierten Geschlechterrollen werden hier als Denkmöglichkeit angeboten bzw. diskutiert. Sie sieht es als eine der wenigen von uns befragten KL nicht als sinnvoll an, Wertefragen eigens zu thematisieren. Denn diese ergäben sich ohnehin aus den Themen Politik und Geschichte (Religionsfreiheit, Diskriminierung, Toleranz, Sozialstaat und viele andere).

In dem von uns besuchten Kurs sind Migrant_innen aus sehr unterschiedlichen Herkunftsländern anzutreffen, die den Kurs überdurchschnittlich gut bewerten. Es werden Themen oder Aktivitäten hervorgehoben, die sie im Rahmen des Kurses noch gerne gemacht hätten, z.B. mehr über Gesetze (Ort1/TN3) bzw. das Grundgesetz (Ort1/TN4) zu erfahren, mehr Exkursionen zu historischen Plätzen zu unternehmen (Ort1/TN1) oder mehr über das Kopftuch zu diskutieren (Ort1/TN2).

Die Kursgestaltung in Ort 1 lässt sich unter dem Begriff ,freiheitlichindividualistisch ' fassen. Der Einstieg in das von uns besuchte Modul erfolgt über den Begriff ,Kultur' und über ,Werte‘ im Allgemeinen. Es werden Ideen dazu gesammelt und diskutiert, was den TN an Deutschland gut gefällt, woran sie sich hingegen schlecht gewöhnen können. Auch die KL beteiligt sich mit eigenen Erfahrungen von Auslandsreisen; es herrscht eine rege Beteiligung und eine fröhliche Stimmung. Die zweite Kurshälfte beschäftigt sich mit „Familie und anderen Lebensgemeinschaften“. Dazu wird ein Bild aus dem Lehrbuch „60 Stunden Deutschland“ (Kilimann et al. 2013: 69) mit unterschiedlichen Familienformen dargeboten. Eine kleine Debatte entspinnt sich zum Adoptionsrecht von Schwulen und Lesben, 
weil sich eine TN diesbezüglich ablehnend zeigt. Ebenfalls kurz diskutiert wird die Vielehe. Ein TN hatte erzählt, dass es in seinem Herkunftsland normal sei, dass ein Mann viele Frauen hat. Auch hier bringt sich die KL ein und weist darauf hin, dass die Vielehe für den Mann mitunter auch sehr stressig sein kann. Kurz besprochen werden am Ende die Gründe, die zur Veränderung der traditionellen Familienstruktur geführt haben: mehr Scheidungen, die Berufstätigkeit von Frauen, Frauenbewegung bzw. Emanzipation, das Prinzip der Individualisierung und persönlichen Freiheit sowie der Wertewandel.

Der nächste Themenblock (zweiter Kurstag) ist Toleranz/Diskriminierung. Die TN lesen gemeinsam den Grundsatz der Gleichbehandlung im deutschen Grundgesetz. Sie sollen einschätzen, ob eine bestimmte Situation oder Handlung als diskriminierend eingestuft werden kann. Hier erweist sich der Text als zu schwer; es entspinnt sich keine Debatte. In einer Gruppenarbeit sollen die TN sodann über von der KL mitgebrachte Bilder diskutieren, die sie als kontrovers bezeichnet. Zwei Bilder werden im Plenum besprochen. Eines zeigt ein Männerpaar mit Kind; für die meisten TN ist dies nicht kontrovers, nur eine TN spricht sich vehement dagegen aus und mehrere TN versuchen, sie vom Gegenteil zu überzeugen. Das andere Bild zeigt eine Gerichtsszene, die sich auf die Klage einer Lehrerin bezieht, in Deutschland mit Kopftuch unterrichten zu dürfen. Die TN drücken dazu vor allem ihr Erstaunen aus, dass Frauen mit Kopftuch in manchen Berufen nicht arbeiten dürfen. Sie sind sich einig, dass es Frauen erlaubt sein solle, sich zu kleiden wie sie wollen. Es geht auch kurz um die Frage, ob ein Kopftuch immer ein religiöses Zeichen sei, dies wird aber nicht vertieft. Die KL resümiert, dass es zu diesem Thema möglicherweise keine letztgültige Meinung gibt, auch sie habe keine gänzlich fixe Position dazu.

Die Frage: Was ist für Sie ,echte 'Gleichberechtigung? Gibt es Gleichberechtigung in Deutschland? wird anhand von fünf Bildern aus dem Lehrbuch diskutiert. Während das Buch anhand eines Bildes von Angela Merkel auf die zunehmende Präsenz von Frauen in der Politik hinweist, kommentiert die KL, dass Angela Merkel oft dazu benutzt wird zu behaupten, dass Frauen heutzutage bereits gleichgestellt seien (Ort1/TB: 7). Das Bild eines Mannes bei der Hausarbeit ist im Buch mit dem Hinweis versehen, wie viele Männer inzwischen bereit seien, sich an der Hausarbeit zu beteiligen. Die KL kommentiert: Hier sieht man, dass nur ein geringer Prozentsatz der deutschen Männer Hausarbeit übernimmt (ebd.). Sie bringt in die Gruppendiskussion über die geschlechtliche Arbeitsteilung den Vorschlag ein, Haus- und Erziehungsarbeit unabhängig vom Geschlecht mit 3000 Euro/netto zu entlohnen (Ort1/TB: 8). Eine abschließende Debatte entspinnt sich zum Thema Lohnschere: Die TN sind über den in Deutschland existierenden Gender-Pay-Gap verwundert und empört. Auf die Frage, ob es denn kein Gesetz zur Lohngleichheit gäbe, antwortet die KL, dass dies nur im öffentlichen Dienst möglich sei, denn 
wenn in der freien Wirtschaft mit Gesetzen eingegriffen würde, dann wäre diese nicht mehr frei (Ort1/TB: 8).

In den Interviews mit den TN in Ort 1 zeigt sich zum Teil eine andere Sicht auf die im Kurs angesprochene egalitäre Geschlechterdifferenz. TN1 argumentiert, dass es einen Unterschied gäbe zwischen Frauen und Männern, der sich spätestens mit Schwangerschaft und Geburt zeige. In ihrer Reflexion über die Kölner Silvesternacht vermutet sie, dass die (derzeitige) Migration die modernen Geschlechterbilder in Deutschland wieder rückgängig mache: Politiker hätten sich geäußert, dass die belästigten Frauen ihren Kleidungsstil anpassen sollten. Das interpretiert sie als einen Backlash hin zu tradierten Modellen von Geschlecht (Ort1/TN1: 12-13). TN1 hat von Köln gehört und drückt ihre Bestürzung über die Vorfälle aus, die sie zunächst mit der Kultur der zugewanderten Täter in Zusammenhang bringt und dann mit deren Arbeitsunwilligkeit. Für sie ist klar: Diese Zuwander_innen wollen die sozialen Sicherungssysteme auszunützen. An anderer Stelle drückt sie ihre Besorgnis über die starke Zuwanderung aus, die schlecht für die kinderschwachen Deutschen sei. Die Deutschen, die als sehr tolerant und unterstützend porträtiert werden, sollten nur solche Menschen aufnehmen, die vor Krieg flüchten - und keine ,Wirtschaftsflüchtlinge‘. Diese Darstellung wird später - möglicherweise weil sie im Konflikt mit ihrer eigenen Arbeitsmigration steht - verändert in eine Entgegensetzung zwischen arbeitswilligen Migrant_innen und jenen, die nur das Sozialsystem ausnutzen wollen (Ort1/TN1: 13-14). TN3 betrachtet die Kölner Vorfälle vorrangig aus der Perspektive seiner weiblichen Verwandten als potenzielle Opfer und führt aus: „Wenn sie sagt: ,Nein“, das einfach Nein. Sie wollte das nicht. [...] Dann auch das habe ich da vorgestellt sofort. Meine Mutter oder meine Schwester oder meine Tochter. Das wie kann ich das vorstellen in mein Kopf. Das finde ich total scheiße.“ (Ort1/TN3: 14) Danach zieht er mögliche Erklärungen in Betracht, etwa das Fehlen ausreichender Information über das Einreiseland, psychische Krankheit oder Kriegstraumata (Ort1/TN3: 14). Alltagsrassismen werden von den TN in Ort 1 ebenfalls erwähnt. Auffällig ist diesbezüglich, dass auf die Benennung direkt eine Relativierung folgt. TN3 wird an einer Busstation beschimpft, er solle Deutschland verlassen, die Öffnung der Grenzen wäre falsch gewesen. Er fügt hinzu: „Aber trotzdem finde ich auch nicht die ganze Leute ist so. Wir manchmal finden nur ein oder zwei Mal Prozent von 100\%.“ (Ort1/TN3: 15) TN4 drückt seine Erfahrungen so aus:

„Weil ich, ich bin, äh, von Afrika. Ich war mit meine Frau. Meine Frau ist eine, ein, von, von hier. Sie ist eine Deutsche. Ähm. Gibt es ein bisschen, also ähm, Diskrimi..., Diskrimination. $\mathrm{Ja}$, aber für mich das ist nicht wichtig, weil ich, ich will nicht Probleme auf der Straße kriegen. Oder. Ich lebe meine Leben und für mich ist. Ja.“ (Ort1/TN4: 13) 
Dass das Demokratie-Lernen und die im Kurs hervorgehobene Chancengleichheit unter strukturell ungleichen Bedingungen stattfinden, wird an Ort 1 besonders darin deutlich, dass die TN eine Einteilung in gute vs. schlechte, Wirtschafts- vs. ,echte Flüchtlinge aufgreifen und über die durch Migration drohende Retraditionalisierung der Geschlechterverhältnisse nachdenken. Das Ereignis ,Köln‘ bleibt als leerer Signifikant zurück, der hierarchische Positionen Migrierter untereinander gestattet. Die Freiheitsversprechen im neuen Land, die im Kurs stark gemacht werden, eröffnen zwar einen utopischen Raum der egalitären Pluralität, machen aber strukturelle Ungleichheitslagen und Rassismuserfahrungen weitgehend unsichtbar.

\subsection{Thematisierung struktureller Benachteiligung}

In Kursort 5 und 7 haben sich zwei Kursleiterinnen freiwillig für ein Interview und eine Teilnehmende Beobachtung gemeldet. Während die erste (Ort5/KL2) sehr interessiert ist an feministischen Diskursen und gleich ein Gespräch darüber beginnt, äußert die KL aus Ort 7, sie wisse nicht, warum sie im Kurs über Geschlechtergerechtigkeit sprechen solle, wo doch auch hierzulande sehr wenig erreicht sei (Ort7/TB: 1). Wie im Verlauf der Interviews und der Beobachtung deutlich wird, beschäftigen sich beide mit gesellschaftstheoretischen Perspektiven auf Geschlechterverhältnisse. Beide befürworten die Erweiterungen des entsprechenden Moduls insbesondere, weil es Ihnen die Möglichkeit verschafft Exkursionen durchzuführen. Die KL aus Ort 7 fügt hinzu, weil Sie ihren Unterricht so gestalten kann, dass er bei den Interessen der Leute ansetzt (Ort7/KL: 1).

Beide gestalten die Kurse sehr unterschiedlich: Die Kursleiterin 2 aus Ort 5 beginnt ihren Unterricht ähnlich wie die KL aus Ort 3 und Ort 4 mit den Bildern zu Familienformen aus dem Lehrbuch Orientierungskurs (Schote 2017: 69). Ihr geht es in diesem Zusammenhang aber nicht nur um die Vermittlung gültiger Regeln und Normen, sondern auch um die Frage, wer in diesen Haushalten die Care-Arbeit übernimmt. Am Beispiel einer alleinerziehenden Mutter erklärt sie, dass Frauen meist dazu angehalten seien, sehr viel Arbeit in dieser Hinsicht zu übernehmen. Die TN sollen sagen, wie es bei Ihnen zu Hause ist. Eine Gruppe von Frauen findet die Verteilung ungerecht, sobald es eine gleichwertige oder bessere Ausbildung gäbe, solle die Arbeit gleich verteilt sein. Zwei Männer sprechen sich dagegen aus: Sie müssten schon so viel arbeiten und würden ungern zuhause noch mehr arbeiten. Zwei andere Männer (einer von ihnen hatte gleich nach der Vorstellungsrunde gesagt, dass er die Benachteiligung von Frauen ablehnt) fordern, dass die Tradition verändert werden müsse. Generell entsteht über den Austausch eine Situation, in der die TN erklären, wie sie ihren Alltag strukturieren und warum das so ist: Ökonomische und strukturelle Bedingungen kommen in den Blick. So hebt der Mann, der nicht putzen will, hervor, dass er derzeit als Putzmann in einer Firma arbeitet 
und in seinem Herkunftsland Ingenieur war; um am Kurs teilzunehmen steht er morgens um fünf auf, putzt und kommt um zwölf Uhr zum Kurs, um ab 17 Uhr weiter zu putzen. Eine andere Frau beschreibt einen ähnlichen Tagesablauf, ebenfalls mit einem Putzjob; sie hingegen muss noch ihre Kinder versorgen. Zum Schluss dieser Sequenz legt die Kursleiterin den Teilnehmenden eine Tabelle zur Aufteilung von Hausarbeit in Deutschland vor, die verdeutlicht, dass Frauen noch immer den höchsten Anteil an Versorgungsarbeit übernehmen.

Im Interview mit der KL von Ort 7 hebt diese zunächst das Spannungsverhältnis hervor, das sich durch den verpflichtenden Charakter der Kurse und die Idee der Wertevermittlung ergibt. Sie sieht es zudem sehr kritisch, ob in der Erwachsenenbildung tatsächlich von Wertevermittlung gesprochen werden kann. Werte, so ist sie überzeugt, werden durch Erfahrungen erlernt und können kaum kognitiv vermittelt erzeugt werden. Auch die kulturelle Differenz, die von anderen KL angesprochen wird, sieht sie nicht als ausschlaggebend. Sie benutzt den Begriff kaum im Unterricht und hebt stattdessen hervor, wie sehr die Menschen, die hier ankommen, vor alltäglichen Herausforderungen stehen - was wenig mit ,Kultur ${ }^{`}$ zu tun habe (Ort7/KL: 2-3).

Im Kurs thematisiert sie auch die Familie, kommt aber relativ schnell auf die Frage der Gleichberechtigung zwischen den Geschlechtern zu sprechen. Um sich diesen Fragen anzunähern nimmt sie im Lehrbuch Mein Leben in Deutschland zwei Seiten vor (Buchwald-Wargenau 2017: 74, 76). Einerseits geht es um familiäre Konflikte und anderseits um die Geschichte feministischer Errungenschaften in Deutschland. Bei der Bildergeschichte zum Thema familiärer Konflikt geht es beispielhaft darum, dass Eltern Einwände gegen den Partybesuch ihrer minderjährigen Tochter haben, gemeinsam dann aber eine Einigung gefunden wird. Die Aufgabe ist es, den Bildern vorgegebene Sätze zuzuordnen. Die Grammatik stellt die TN hier vor eine kaum zu bewältigende Herausforderung. Trotzdem kommen sie auf eigene Erfahrungen zu sprechen und heben hervor, dass sie Konflikte mit ihren Kindern nicht gewaltsam austragen. Nachdem alle darüber gesprochen haben, wie Konfliktaustragung ideal funktionieren sollte und hervorgehoben hatten, dass alle gleichberechtigt seien, sagt die KL - etwas ironisch - ,ja auf dem Papier“ (Ort7/TB: 2).

Um ihren Einwand zu illustrieren, fordert sie die TN auf, die nächste Seite im Buch aufzuschlagen: Es geht um die Geschichte der Frauenbewegung. Thematisiert wird das Wahlrecht, das Recht auf Bildung, die Verteilung von Care-Arbeit, Vergewaltigung in der Ehe und die Wahl der ersten weiblichen Bundeskanzlerin. Auch diese Übung zielt auf Leseverständnis und nur in zweiter Hinsicht auf eine inhaltliche Auseinandersetzung. Da der Text schwierig ist, entspinnt sich keine Diskussion, aber die TN sind sehr verwundert über die gesetzlichen und politischen Verhältnisse in Punkto Gleichstellung in Deutschland. Im Anschluss an diese Übung erzählen die weiblichen TN im Interview, wie die Frauenbewegung in ihrem Her- 
kunftsland $\mathrm{Fu} ß$ gefasst habe und welche Änderungen hierdurch initiiert wurden (vgl. TN2 u.3/Ort7: 3).

Obwohl beide KL ein gänzlich differentes Herangehen haben, setzen sie beide auf die Thematisierung struktureller Problematiken (und auf die Diskussion von Lösungsideen) in Deutschland. In beiden Unterrichtseinheiten herrschte eine lockere und interessierte Atmosphäre, die Teilnehmenden gaben zu Protokoll, dass sie sich in den Kursen gut aufgehoben fühlten, obwohl auch sie von teilweise massiven Alltagsrassismen außerhalb des Kurses berichteten. TN3/Ort7 erzählte beispielsweise, dass seine Nachbarin ihn über den Zaun hinweg als Flüchtling beschimpfen würde (vgl. TN3/Ort7: 1).

Im Unterschied zu anderen Kursorten wird in Ort 3 die Erweiterung aller Module des Kurses, also auch des politischen und historischen Teils, hervorgehoben, den die Aufstockung auf 100 Stunden ermögliche. In Bezug auf wichtiges Orientierungswissen werden hier weniger die Alltagskommunikation und Wertefragen, sondern Unterkunft, Arbeit und Behördengänge ins Zentrum gerückt, mit denen die Menschen beschäftigt seien (Ort3/OE: 6, 11). KL1 dieses Kursortes ist die Vermittlung historischen Wissens besonders wichtig, denn ohne dieses ist, wie sie betont, die deutsche Gegenwart gar nicht zu verstehen. Darüber hinaus legt sie ihren Schwerpunkt auf die Themen Demokratie, Verfassung und Freiheit sowie auf eine machtkritisch verstandene Interkulturalität, ein Thema, mit dem sie sich intensiv befasst hat.

OE und KL1 betonen Strukturen der Benachteiligung von Migrant_innen und zeigen auf, dass Fragen des Wohnens und Arbeitens sowie stärkere Kontakte zu Deutschen sehr viel wichtiger wären als jedes, Kulturlernen'. KL1 bemüht sich in ihrer Kurspraxis um einen Austausch der TN untereinander; Wissensvermittlung und Reflexion sind für sie wichtige Methoden. Sie weist auf die starken Zwänge hin, unter denen die TN stehen und die es ihnen oftmals erschweren würden, vom Kurs zu profitieren. Insbesondere fehle es am Austausch mit der Mehrheitsgesellschaft, was vor allem bei Ausflügen und in den Pausengesprächen deutlich werde. Als Kursleiterin ist es ihr Anliegen, politische und geschichtliche Informationen zu vermitteln, aber nicht, die Menschen zu läutern oder zu verändern (Ort3/KL1: 11). Sie möchte Menschen dafür sensibilisieren, wie es ist, hier zu leben, welche Gesetze es gebe, und für die Bedeutung von Toleranz und Freiheit. Die Themen Homosexualität und die ,Ehe für alle' seien manchmal schwierige Themen im Kurs, allerdings eher von TN aus den EU-Ländern. Jene, denen im öffentlichen Diskurs diesbezüglich konservative bzw. intolerante Meinungen zugeschrieben werden, äußerten sich zu diesen Themen oftmals kaum (Ort3/KL1: 5).

Angesprochen auf die Ereignisse in Köln wird auch an Ort 3 eine Abgrenzung zu den ,neuen' Migrationsgruppen deutlich. Das zeigt sich in den Gesprächen durch Verweise darauf, dass sich die hier versammelten Migranten von den Tätern von Köln unterscheiden würden. Die an diesem Standort interviewten TN - die zumeist 
aus dem europäischen Raum kommen - machen darauf aufmerksam, dass die Täter nicht aus der EU kamen. Menschen aus diesen Kulturkreisen bräuchten eine Reflexion über Geschlechterverhältnisse und Rollenverständnisse, während dies für sie selbst bereits bekanntes Wissen sei. Grundsätzlich messen alle TN der Gleichberechtigung zwischen Männern und Frauen große Bedeutung bei. „Männer und Frauen sind gleich“, betont insbesondere TN2, ,ich finde, das muss immer sein.“ (Ort 3/TN2: 7) Er weist auf den Zusammenhang von Kultur und Geschlechterverhältnissen hin und betont, dass seine Herkunftskultur der deutschen Kultur sehr ähnlich sei.

Auffällig ist, dass die Thematisierung der Geschlechtergleichstellung im Kurs 1 an Ort 3 weniger an der ,modernen' deutschen Kultur festgemacht wird, sondern an erkämpften politischen Veränderungen. Diese Herangehensweise ermöglicht eine andere Thematisierung hierarchischer Strukturen und gesellschaftlicher Benachteiligungen. Der Zugang von KL1 nutzt historische Beispiele für politische Diskussionen und Meinungsaustausch, die viel Raum für die Erfahrungen der TN lassen. Sowohl KL1 als auch die Programmverantwortliche bringen Begriffen wie (Leit-) Kultur oder einseitigen Integrationsforderungen Skepsis entgegen, sie verdeutlichen darüber hinaus Ambivalenzen in der Kursgestaltung: Der Kurs stellt zum einen historisches und politisches Wissen zu Verfügung, ist zum anderen aber auch eine Gratwanderung, nämlich zwischen Interesse und Respekt vor dem, was die Menschen mitbringen und den Informationen darüber, was in Deutschland als normal gilt oder von ihnen erwartet wird (Ort3/KL1: 9).

\subsection{Zwischenfazit: Effekte gesellschaftlicher Diskurse auf die soziale Praxis von Orientierungskursen}

Wie ist nun das Verhältnis zwischen den gesellschaftlich-medialen Diskursen am Kreuzungspunkt von Antifeminismus und Rassifizierung und der sozialen Praxis von Orientierungskursen zu denken? Inwiefern finden sich Fragmente der gesellschaftlichen Diskurse auch in der sozialen Praxis wieder? Der Blick auf die Sichtweisen von Kursbeteiligten, auf die Curricula und Lehrwerke sowie auf die soziale Praxis in den Kursen zeigt: Es gibt keinen direkten Transfer antifeministischrassistischer Diskurse in die Praxis der Integrationskurse. Insbesondere Versatzstücke aus dem ersten von uns identifizierten gesellschaftlich-medialen Diskurs, dem bevölkerungspolitischen Diskurs, haben hier keinerlei Platz. Sehr deutlich betonen die von uns befragten Programmverantwortlichen und Kursleitungen, dass sie die Kurse als Orte des Demokratielernens, der Freiheit, Gleichheit und Partizipation verstehen und damit auch als Orte, an denen Geschlechtergerechtigkeit, Toleranz und Pluralität als bedeutsame Ideale für das Zusammenleben thematisiert und dis- 
kutiert werden. In Bezug auf den Überlegenheits-Diskurs und den Gefährdungs-/ Verführungsdiskurs kommen wir jedoch zu einer differenzierteren Antwort.

In seiner starken Variante diskreditiert der Überlegenheitsdiskurs gesellschaftspolitische Bemühungen um Vielfalt, nutzt aber bestimmte frauenpolitische Errungenschaften für eine abwertende und pauschale Entgegensetzung zu jenen, die jenseits solcher Errungenschaften verortet werden. Anhand der Interviews und mit Blick auf die Kurspraxis zeigt sich, dass sowohl den Programmverantwortlichen und den Kursleitungen als auch den Teilnehmenden Geschlechtergerechtigkeit und die Anerkennung von Vielfalt wichtige Anliegen sind. Verunglimpfungen und Herabsetzungen aufgrund von Geschlecht, Herkunft oder Sexualität finden nicht nur keinen Platz im Kursgeschehen, sie werden in den Interviews von KL und OE explizit als Widerspruch zum grundlegenden Zugang in den Kursen zurückgewiesen. Unserer Interpretation der Daten zufolge finden zwei Dimensionen des Überlegenheitsdiskurses im Kursgeschehen dennoch Widerhall, allerdings in einer vermittelten und (mehr oder weniger) subtilen Form.

Zum einen ist davon auszugehen, dass die Erweiterung des Kurses von bisher 60 auf 100 Stunden eine Reaktion auf die im öffentlichen Diskurs geäußerte Sorge um starke kulturelle Differenzen eines großen Teils der seit 2015 Geflüchteten und neu Zugewanderten zur deutschen Mehrheitsgesellschaft darstellt - und zwar insbesondere in Bezug auf Geschlechtervorstellungen. Diese Interpretation stützt sich zum einen auf die Interviews: Die Kurse werden von Programmverantwortlichen vereinzelt als Orte wahrgenommen, die solchen Geschlechterproblematiken entgegenarbeiten können. Die OE in Ort 1 sieht ,Köln` diesbezüglich nur als Kristallisationspunkt von Problemen, die bereits vorher rund um den Kurs deutlich waren. Zum anderen stützen wir uns auf Veränderungen im Curriculum und in den Kursmaterialien. Im neuen, auf 100 Stunden erweiterten Curriculum spiegelt sich das Anliegen, in Bezug auf Geschlechterverhältnisse, (Homo-)Sexualität und Toleranz starken Unterschieden Rechnung zu tragen und dem Auftauchen von Problemen und Missverständnissen entgegenzuwirken. Blickt man etwa auf die Erweiterung der Kursunterlagen des Klett-Verlag, so lassen sich im Vergleich des Vorgängerbuchs 60 Stunden Deutschland (Kilimann et al. 2013) mit dem neuen Lehrbuch 100 Stunden Deutschland (Butler et al. 2017) unschwer jene Themen wiederfinden, die auch die öffentliche Debatte in Bezug auf Geschlecht am stärksten beschäftigten, insbesondere Verhaltensregeln in öffentlichen Schwimmbädern und/oder in der Schule, das Thema der weiblichen Bekleidung, die (vermeintliche) Selbstverständlichkeit von Karrieremöglichkeiten, Berufsvielfalt und öffentlichen Aktivitäten von Frauen in Deutschland sowie die geschlechtliche Arbeitsteilung in Haushalt und Kinderbetreuung. In unseren Gesprächen trafen wir allerdings auch auf Vorbehalte oder Skepsis, was diese curricularen Schwerpunktsetzungen betrifft.

Mit Blick auf den Gefährdungs-/Verführungsdiskurs lässt sich sagen, dass die Neuakzentuierung der Kurse eine männliche, in Sachen Geschlechtergleichheit und 
-freiheit defizitäre Zielgruppe adressiert. So findet insbesondere der Gefährdungsaspekt im Kursgeschehen Beachtung; häufig in Form von ambivalenten Bezugnahmen und Distanzierungen von den ,Kölner Tätern“ durch die Kursbeteiligten. Der Gefährdungsdiskurs hat also im Kursgeschehen durchaus Spuren hinterlassen - insbesondere bei jenen Teilnehmern, die aufgrund ihres äußeren Erscheinungsbildes davon ausgehen müssen, dass sie mit der rassifizierten Gruppe der , arabischen Anderen' identifiziert werden. Bei diesen Teilnehmern fanden sich jedoch auch andere Thematisierungen, wie oben deutlich wurde, etwa wenn ein TN die Perspektive seiner weiblichen Verwandten einnimmt und das Einspruchsrecht von Frauen hervorhebt (TN3/Ort1).

\section{Gesellschaftstheoretische Reflexion DER VERSCHRÄNKUNG VON GESCHLECHT UND RACE}

Im Folgenden möchten wir unsere Blickrichtung ändern und die oben dargelegten Befunde zu den Orientierungskursen vor dem Hintergrund gesellschaftstheoretischer Überlegungen zum Status quo des gegenwärtigen Geschlechter- und Migrationsregimes reflektieren. Auch wenn die Orientierungskurse dabei unser Referenzpunkt bleiben, möchten wir unsere Befunde zu vier Thesen verdichten, um die herausgehobene Rolle von Geschlecht und race in beiden von uns anvisierten Kräftefeldern besser zu verstehen.

\subsection{Adressierung als kulturelle versus als politische Subjekte}

Unsere erste These lautet: Die Einladung zur Teilhabe am demokratischen Prozess adressiert die Teilnehmenden stärker als kulturelle denn als politische Subjekte. Geschlecht und race werden damit zu kulturalisierten Differenzen, die im pluralen Nebeneinander von Meinungen und Positionen ihren neutralisierten Platz finden.

Prominent genannte Ziele von Seiten der Programmverantwortlichen und Kursleiter_innen sind gegenseitige Toleranz, Offenheit, Empathiefähigkeit, Meinungspluralität sowie die Einladung, an Freiheit und Gleichheit in Deutschland zu partizipieren. Dieses wichtige Ziel wird von der Mehrheit der KL keineswegs ethnisierend oder rassifizierend formuliert, im Gegenteil: Wie KL an Ort 1 und Ort 3 betonen, wäre der Kurs für alle in Deutschland Lebenden ein Gewinn, weil er grundlegendes staatsbürgerliches Wissen vermittele, das in der Mehrheitsgesellschaft zweifellos stark lückenhaft sei. Aber auch das kulturelle Miteinander und die Kooperation im Kurs wird hervorgehoben, als Beispiel gelebter Demokratie und Pluralität. Gerade von jenen KL, die Vereinseitigungen und Versämtlichungen in Bezug auf ethnisch geprägte Vorstellungen bzw. Einstellungen kritisch reflektieren, wird dem 
Fokus auf Meinungsbildung, Meinungsaustausch und Meinungsvielfalt großes Gewicht eingeräumt.

An diesem zentralen Ziel und seiner Umsetzung wird allerdings eine wichtige Verschiebung deutlich: Denn die Teilnehmenden werden zwar zur Partizipation an und Einübung von demokratischen Praxen eingeladen und ermutigt - allerdings weniger als politische denn als kulturelle Subjekte. Die Reflexionen, Diskussionen und der Meinungsaustausch, die im Kurs für die Umsetzung dieses Ziels vorgesehen sind, zielen insbesondere auf die wechselseitige Anerkennung als kulturelle Andere. In diesem Sinne ist der Kurs neben der Wissensvermittlung in Bezug auf Rechte, Pflichten und Gepflogenheiten in Deutschland sehr stark als interkultureller Begegnungsraum konzipiert, der von politischen Fragestellungen freizuhalten ist. Die Verschiebung auf die kulturelle Ebene - auf Fragen der Wertedifferenzen und Wertepluralität - scheint eine Abfederung des Spannungsverhältnisses zwischen dem Auftrag, über Gegebenheiten des Aufnahmelandes zu informieren, und dem politischen Bildungsauftrag im Sinne der Einladung zur aktiven Partizipation durch Mündigkeit, Reflexion und Meinungsfreiheit darzustellen, in dem sich die Orientierungskurse befinden.

Dieses Spannungsverhältnis beschreibt Rubia Salgado (2015: 133) folgendermaßen: „Einerseits sollen die Lernenden den Bildungsprozess ausgehend von ihren jeweiligen gesellschaftlichen Positionen, von ihren spezifischen Geschichten, Erfahrungen, Wissen, Werten und Normen gestalten; andererseits sollen sie sich auch Normen, Werte und Wissen der Dominanzgesellschaft aneignen“. Diese doppelte Orientierung benennt sie deutlich als Herausforderung, die weder in die eine noch in die andere Richtung aufgelöst werden kann oder sollte, sondern als Dissonanz aufrechterhalten werden muss. Die Kulturalisierung von Unterschieden übernimmt diesbezüglich allerdings die Funktion, gesellschaftliche Ungleichheitsverhältnisse unsichtbar zu machen. Diese können somit nicht zu einem Bestandteil des Lernund Lehrprozesses oder zu einem Herzstück des Demokratielernens werden.

\subsection{Diversity-Sensibilität und Postfeminismus}

Unsere zweite These lautet: Die Rahmung und Thematisierung von Geschlechterfragen als moderne Rollenvielfalt macht feministische Kämpfe der Gegenwart unsichtbar, verdunkelt strukturelle Barrieren im Ergreifen von Karrierechancen und verwandelt gesamtgesellschaftliche Probleme der Hierarchie im Geschlechterverhältnis, im Migrationsregime und in Form gegenwärtiger Rassismen in Fragen der individuellen Selbstverantwortung.

Die curriculare Einbettung von Geschlecht, Sexualität, Kultur und Herkunft und ihre Thematisierung in den Lehrbüchern des Kurses folgt weitgehend einem Verständnis von Vielfalt und Diversität, das von seinem Bezug zu strukturellen Macht- 
unterschieden losgelöst ist. Im Zentrum steht zum einen die Reflexion von und Toleranz gegenüber unterschiedlich ausgestalteten Lebensformen; zum anderen zielen die Diskussionsangebote schwerpunktmäßig auf die Arbeitsmarktintegration von Frauen und den Abbau diesbezüglicher Hindernisse, die vorrangig als veraltete Rollenerwartungen und überkommene Klischees gefasst werden.

In den Lehrbüchern finden sich dazu zahlreiche Beispiele, die von den Teilnehmenden reflektiert und in Frage gestellt werden sollen, etwa klassische Zuschreibungen an Mädchen und Jungen in Bezug auf Farben, Spielzeug, Aktivitäten, Fähigkeiten und Vorlieben, die sich in geschlechtsspezifischen Erziehungsstilen niederschlagen können. Frauen und Männer werden in ,untypischen' Berufsfeldern gezeigt: etwa Frauen auf dem Bau, bei der Polizei, in der Politik und Männer als Erzieher oder als (vorübergehende) Hausmänner. Besondere Aufmerksamkeit kommt aber dem als veraltet präsentierten Modell von Hausfrau und Alleinverdiener zu, dem das zeitgemäße Modell des Doppelverdienerhaushalts gegenübergestellt wird.

Unsere Analyse der Orientierungskurse macht deutlich, dass sich der Fokus auf Diversität sehr gut dafür einsetzen lässt, Verhältnisbegriffe wie Geschlecht, race, Klasse oder Nationalität auf Fragen der individuellen Haltung und des durch Reflexion überwindbaren Vorurteils zu verengen. Damit lassen sich über diesen Begriff neoliberale Optimierungsforderungen mit marktwirtschaftlichen Erwägungen und Freiheitsversprechen verschmelzen. Gender kann auf diese Weise jenseits der strukturellen Geschlechterhierarchie angesprochen werden und in eine individuell bzw. familiär handhabbare Angelegenheit verwandelt werden. Eine Ausnahme bildet diesbezüglich die Thematisierung der geschlechtsspezifischen Lohnungleichheit ${ }^{5}$.

Diese Thematisierungsweise lässt sich auch mit dem Begriff des Postfeminismus einfangen. Sie ist von der Grundannahme getragen, dass Geschlechtergleichheit oder -gerechtigkeit in Deutschland weitgehend erreicht seien - auch wenn sich letzte Schönheitsfehler wie Lohnungleichheit oder veraltete Klischees auffinden lassen. Beides aber, so die Suggestion, wird sich durch den bereits eingeschlagenen Weg beseitigen lassen. Ohne Thematisierung der sozialen und politischen Kämpfe, die dem Status quo vorausgegangen sind, insbesondere aber ohne Thematisierung der weiterhin bestehenden Geschlechterhierarchie auf dem Arbeitsmarkt, in der Sorgearbeit, in der Einkommensverteilung, in Bezug auf sexualisierte Gewalt und in gesellschaftlich relevanten Entscheidungsfunktionen wird die Notwendigkeit

5 Diese wird von einigen Kursleiter_innen explizit thematisiert und auch in einzelnen Lehrbüchern aufgegriffen. Etwa in 100 Stunden Deutschland, wo sie zum einen mit der häufigen Teilzeitarbeit von Frauen, zum anderen mit einer nicht näher bestimmten Diskriminierung erklärt wird, die in der Gesellschaft noch vorzufinden sei (Butler et al. 2017: 102). 
weitergehender Forderungen und kollektiver Kämpfe negiert. Stattdessen bietet die als Modernisierung angebotene Rollendiversität ,postfeministische Ersatzidentifikationen“" (McRobbie 2010: 50) an - auch wenn diese nicht, wie bei McRobbie, den Aspekt der Konsumkultur betonen (müssen), sondern vor allem das Ideal individueller Handhabbarkeit von Freiheit und Selbstverwirklichung ins Zentrum rücken: Alle hier lebenden Individuen sind eingeladen, die sich ihnen bietenden Chancen zu ergreifen und diese ihren Wünschen entsprechend zu gestalten.

Im Kontext des gegenwärtigen Migrationsregimes erhält die postfeministische Färbung von Geschlechterfragen eine zusätzliche Wendung. Die Rahmung geschlechtlicher Vielfalt als kulturelle Errungenschaft kontrastiert mit erheblichen Barrieren in Bezug auf Arbeitsmarktchancen für (bestimmte) Migrant_innen.

\subsection{Staatliche Bildungsinstitutionen im Lichte wohlfahrtsstaatlicher Transformation}

Unsere dritte These lautet: In Orientierungskursen als staatlichen Bildungsinstitutionen zeigt sich eine bestimmte Form der Pädagogisierung des gegenwärtigen Umbaus des Wohlfahrtsstaates.

In der Geschlechterforschung ist wiederholt darauf hingewiesen worden, dass sich die Transformation von fordistischen zu postfordistischen Gesellschaften und die damit verbundene Transformation des Wohlfahrtsstaates höchst geschlechtsspezifisch vollzieht. In diesem Wandlungsprozess zeigt sich in Bezug auf Geschlechterverhältnisse eine komplexe Gleichzeitigkeit von Retraditionalisierung und Flexibilisierung/Modernisierung, die häufig unter den Stichworten Persistenz und Wandel diskutiert wird (Hausotter 2010; Auth/Buchholz/Janczyk 2010; Casale/Forster 2011; Rendtorff/Riegraf/Mahs 2019). Die Liberalisierung der Geschlechterordnung hat sich dabei für Frauen als zweischneidig erwiesen. Zum einen konnten langjährige politische Forderungen nach einem Ende der Abhängigkeit von einem männlichen Ernährer, nach Öffnung des Arbeitsmarktes und der Entnormierung von Geschlechterbildern aufgegriffen und umgesetzt werden. Andererseits führt die spezifische Neubestimmung des Verhältnisses von Staat, Ökonomie und Geschlecht nicht dazu, Sorgearbeit zu einer gesamtgesellschaftlichen Verantwortung zu machen, im Gegenteil: Die Haus-, Erziehungs- und Pflegearbeit muss nun entweder ,nebenbei' erledigt oder in Form prekarisierter Dienstleistungen an andere Frauen (meist an Migrantinnen) weitergegeben werden. Die Liberalisierung der Geschlechterverhältnisse führt daher zu Mehrfachbelastungen oder zu einer rassifizierten Unterschichtung des Privathaushalts (Lutz 2007; Haidinger/Knittler 2016). Frauen werden somit als geschlechtsneutrale Marktteilnehmer_innen adressiert, während in Bezug auf Reproduktionsarbeit eine Re-Aktualisierung von Geschlecht stattfindet (Soiland 2009). 
Im Prozess der gegenwärtigen Transformation übernehmen Bildung und Erziehungsinstitutionen wichtige Funktionen. Oft bemühte Slogans wie ,Investition in Bildung', ,Bildung als Ressource', so Casale/Forster (2011), legen Zeugnis vom zunehmenden Fokus auf eine Investition in Humankapital ab - Frauen und in unserem Fall Zuwanderer_innen werden dabei als ökonomisch wertvolle Ressource erkannt.

Wir schlagen vor, Orientierungskurse auch im Hinblick auf ihre Funktion für die Aktivierung von Humanressourcen für die umgebaute (entsolidarisierte) Wohlfahrtsgesellschaft zu analysieren. Somit stellt sich die Frage, inwieweit die Sensibilisierung für Geschlechterrollen und sexuelle Freiheit, für Diversität und Kultur hier Vereinnahmungen ursprünglich herrschaftskritischer Forderungen für neoliberale Macht- und Herrschaftsverhältnisse erlauben. Der Bezug auf kulturelle oder Wertefragen und eine Engführung von Geschlecht auf individuelle Vorurteile und Haltungen bietet zumindest eine Ausgangsfolie für die Dethematisierung sozialer Fragen und gesellschaftlicher Konfliktdynamiken. Paradoxerweise stehen die Genderund Diversitätssensibilisierungen, die im Kursgeschehen Platz finden, auf diese Weise für ein Unsichtbarwerden bzw. -bleiben von Rassifizierung und Geschlechterhierarchie.

Warum aber, so bleibt zu fragen, sind es gerade die außereuropäischen, im Moment zumeist muslimischen Anderen, die als ,Störenfriede‘ des Fortschrittsnarrativ auftauchen? Warum werden im aktuellen Transformationsprozess (de-)politisierte Themen rund um Geschlecht und Sexualität bedeutsam, während race als Strukturkategorie dethematisiert wird? Einerseits ließe sich dazu sagen, dass Europa insgesamt durch eine spezifische Desartikulation von race geprägt ist - Boulila (2019) spricht von einer „racelessness“, in der die Kategorie sichtbar und unsichtbar zugleich ist; andererseits wird race durch spezifische Thematisierungsweisen von Geschlecht auch hervorgebracht.

Zum anderen müsste die Thematisierung von Geschlecht und Sexualität - die, wie wir betont haben, im Orientierungskurs keinerlei Spielraum für Antifeminismus oder Anti-Genderismus eröffnet, sondern vielmehr Wertschätzung und Toleranz gegenüber geschlechtlicher und sexueller Diversität betont - mit weiterführenden Fragen konfrontiert werden. Denn durch ihre Entkoppelung von gesellschaftlichen Macht- und Herrschaftsverhältnissen, insbesondere vom Beharrungsvermögen der Geschlechterhierarchie und der Rassifizierung, läuft sie Gefahr, selbst zu einem herrschaftlichen Instrument zu werden.

\subsection{Race und die strukturelle Dimension des Migrationsregimes}

Unsere vierte These besagt: Wenn sich Zuschreibungen und strukturelle Nachteile häufig im Kontext von ethnischen, rassifizierenden, religiösen, aber in jedem Fall sichtbaren Merkmalen äußern, dann würde es sich lohnen eine kritisch-analytische 
Perspektive entlang rassifizierter Merkmale zu entwickeln und von race und nicht von Migration zu sprechen.

Ausgangspunkt dieser Überlegungen sind Fragen und Analysen, die zuletzt in der kritischen Migrationsforschung angesprochen wurden (Goldberg 2006; Gutiérrez Rodríguez 2018; Boulila 2019). Obwohl in Deutschland sowohl der Begriff Rassismus als auch der damit zunächst verbundene Begriff der Rasse als überkommen gilt, mit dem Nationalsozialismus in Verbindung gebracht und damit gern als ,überwunden' betrachtet wird (vgl. z.B. El-Tayeb 2016), weisen einige Wissenschaftler_innen darauf hin, dass das dieser Kategorisierung zugrunde liegende Denken in keinster Weise überwunden sei, im Gegenteil: die strukturelle Benachteiligung hat nach wie vor Bestand.

„For Europeans, race is not, or really is no longer. European racial denial concerns wanting race in the wake of WorldWar II categorically to implode, to erase itself. This is a wishful evaporation never quite enacted, never satisfied. A desire at once frustrated and displaced, racist implications always lingering and diffuse, silenced but assumed, always already returned and haunting, buried but alive. Race in Europe has left odourless traces but ones suffocating in the wake of their at once denied risinous stench." (Goldberg 2006: 334)

Der Nationalsozialismus als Referenzpunkt von race ruft eine spezifische Perspektive auf. Vergessen ist der koloniale Hintergrund Europas; vergessen auch die damit verbundene Kontinuität: Im Kolonialismus wurden bereits Instrumentarien und Handlungsweisen angewandt, die im Nationalsozialismus Verwendung fanden. „This stress on the Holocaust, however, does account for what Stuart Hall [...] has characterized as historical amnesia, the now deafening silence in Europe concerning its colonial legacy.“ (Ebd.) Diese colonial legacy ist im gegenwärtigen Migrationsregime noch immer präsent und zeigt sich nicht nur und in erster Linie an der Art der Zuschreibungen, Übergriffe und Gewalterfahrungen, sondern gerade auch in ökonomischen Strukturen (vgl. Georgi 2018), in institutionellen Benachteiligungen und in der staatlichen Verwaltung von Migration.

\section{LITERATUR}

Ahmed, Leila (1992): Women and Gender in Islam. Historical Roots of a Modern Debate, New Haven: Yale University Press.

AK Fe.In (Götz, Judith/Berg, Anna/Sanders, Eike) (2019): Frauen*rechte und Frauen*hass: Antifeminismus und die Ethnisierung von Gewalt, Berlin: Verbrecher Verlag. 
Auth, Diana/Buchholz, Eva/Janczyk, Stefanie (2010): Selektive Emanzipation Analysen zur Gleichstellungs- und Familienpolitik, Opladen/Farmington Hills: Barbara Budrich.

Balibar, Étienne (1992): „Gibt es einen ,Neo-Rassismus“?“, in: Étienne Balibar/ Immanuel Wallerstein (Hg.), Rasse, Klasse, Nation. Ambivalente Identitäten, Hamburg: Argument, S. 23-38.

von Braun, Christina/Mathes, Bettina (2007): Verschleierte Wirklichkeit. Die Frau, der Islam und der Westen, Berlin: Aufbau Verlag.

Botsch, Gideon/Kopke, Christoph (2018): „Der ,Volkstod“. Zur Kontinuität einer extrem rechten Paranoia“, in: Juliane Lang/Ulrich Peters (Hg.), Antifeminismus in Bewegung. Aktuelle Debatten um Geschlecht und sexuelle Vielfalt, Hamburg: Marta Press, S. 63-90.

Boulila, Stefanie (2019): Race in Post-racial Europe: An Intersectional Analysis, London: Rowman and Littlefield International.

Boulila, Stefanie/Carri, Christiane (2017): „On Cologne: Gender, Migration and Unacknowledged Racisms in Germany“, in: European Journal of Women's Studies 24 (3), S. 286-293.

Casale, Rita/Forster, Edgar (2011): Ungleiche Geschlechtergleichheit. Geschlechterpolitik und Theorien des Humankapitals. Jahrbuch Frauen und Geschlechterforschung in der Erziehungswissenschaft, Folge 7/2011, Opladen: Barbara Budrich.

Clemm, Christina/Hark, Sabine (2016): „Sind wir über Nacht zu einer feministischen Nation geworden?“, in: Die Zeit vom 18.01.2016. [https://www.zeit.de/ kultur/2016-01/feminismus-uebergriffe-koeln-clemm-hark-10-nach-8; abgerufen am 20.03.2020].

Dieterich, Manuel (2016): „Das seh ich erst, wenn ich's glaube. Das Paradigma kultureller Differenz am Beispiel des Diskurses über die Ereignisse am Kölner Hauptbahnhof in der Silvesternacht 15/16“, in: Soziologieblog vom 26.09.2016. [https://soziologieblog.hypotheses.org/9815; abgerufen am 20.03.2020].

Dietze, Gabriele (2016): „Das Ereignis Köln“, in: Femina Politica - Zeitschrift für feministische Politikwissenschaft 25 (1), S. 93-102.

Dietze, Gabriele (2017): Sexualpolitik. Verflechtungen von Race und Gender, Frankfurt a.M.: Campus.

Dziuba-Kaiser, Stephanie/Rott, Janina (2016): „Immer eine Armlänge Abstand vom ,Anderen“? Zur medialen Berichterstattung über das ,Ereignis Köln““, in: Femina Politica - Zeitschrift für feministische Politikwissenschaft 25 (2), S. 121129.

El-Tayeb, Fatima (2016): Undeutsch. Die Konstruktion des Anderen in der postmigrantischen Gesellschaft, Bielefeld: transcript.

Fanon, Frantz (2008): Black Skin, white Masks, New York: Grove Press.

Farris, Sara (2011): „Die politische Ökonomie des Femonationalismus“, in: Feministische Studien 29 (2), S. 321-334. 
Fritzsche, Christopher (2017): „,Wir respektieren Frauen (und wollen wieder Männer sein).' Geschlechtspolitische Diskurse in der neurechten Wochenzeitung ,Junge Freiheit‘ nach den sexuellen Übergriffen in der Kölner Silvesternacht 2015/16“, in: FJSB+plus. Online-Supplement des Forschungsjournals Soziale Bewegungen 3 (2) [http://forschungsjournal.de/sites/default/files/fjsbplus/fjsbplus_2017-2_fritzsche.pdf; abgerufen am 20.03.2020].

Ganz, Katrin/Meßmer, Anna-Katharina (2015): „Anti-Genderismus im Internet. Digitale Öffentlichkeiten als Labor eines neuen Kulturkampfes“, in: Hark/Villa, Anti-Genderismus. Sexualität und Geschlecht als Schauplätze aktueller politischer Auseinandersetzungen, S. 59-77.

Georgi, Fabian (2018): Managing Migration? Eine kritische Geschichte der Internationalen Organisation für Migration (IOM), Berlin: Bertz+Fischer Verlag.

Gesterkamp, Thomas (2010): Geschlechterkampf von rechts - Wie Männerrechtler und Familienfundamentalisten sich gegen das Feindbild Feminismus radikalisieren. Expertise der Friedrich-Ebert-Stiftung. [https://library.fes.de/pdf-files/ wiso/07054.pdf; abgerufen am 20.03.2020].

Gill, Rosalind (2018): „Die Widersprüche verstehen. (Anti-)Feminismus, Postfeminismus, Neoliberalismus“, in: Aus Politik und Zeitgeschichte 68 (17), S. 12-19.

Goldberg, David Theo (2006): „Racial Europeanization“, in: Ethnic and Racial Studies 29 (2), S. 331-364.

Grubner, Barbara (2016): „Freiheit, Pluralität und politische Urteilskraft. Überlegungen zu feministischer Bündnispolitik jenseits der vergeschlechtlichten Kulturdebatte der Gegenwart", in: Barbara Grubner/Carmen Birkle/Annette Henninger (Hg.), Feminismus und Freiheit. Geschlechterkritische Neuaneignungen eines umkämpften Begriffs, Sulzbach: Ulrike Helmer, S. 236-263.

Gutiérrez Rodríguez, Encarnación (1997): „Jenseits des Integrations- und Differenzdiskurses - zur Frage von Subjektpositionen und Verhandlungsräumen von Migrantinnen in der BRD“, in: Karl-Siegbert Rehberg (Hg.), Differenz und Integration: die Zukunft moderner Gesellschaften. Verhandlungen des 28. Kongresses der Deutschen Gesellschaft für Soziologie im Oktober 1996 in Dresden. Band 2: Sektionen, Arbeitsgruppen, Foren, Fedor-Stepun-Tagung, Opladen: Westdt. Verl., S. 136-141 [https://nbn-resolving.org/urn:nbn:de:0168-ssoar-13 9339; abgerufen am 09.04.2020].

Gutiérrez Rodríguez, Encarnación (2016): „,Flüchtlingskrise‘, Kolonialität und Rassismus - eine andere Grammatik der Krise des Kapitalismus denken“, in: Das Argument 319/2016, S. 669-682.

Gutiérrez Rodríguez, Encarnación (2018): „The Coloniality of Migration and the ,Refugee Crisis': On the Asylum-Migration Nexus, the Transatlantic White European Settler Colonialism-Migration and Racial Capitalism“, in: Refuge: Canada's Journal on Refugees 34 (1), S. 16-28. 
Gutiérrez Rodríguez, Encarnación/Tuzcu, Pinar/Winkel, Heidemarie (2018): „Introduction: Feminisms in Times of Anti-Genderism, Racism and Austerity“, in: Women's Studies International Forum 68, S. 139-141.

Haidinger, Bettina/Knittler, Käthe (2016): Feministische Ökonomie. Eine Einführung, Wien: Mandelbaum.

Hark, Sabine/Villa, Paula Irene (2015): Anti-Genderismus. Sexualität und Geschlecht als Schauplätze aktueller politischer Auseinandersetzungen, Bielefeld: transcript.

Hark, Sabine/Villa, Paula Irene (2017): Unterscheiden und herrschen, Bielefeld: transcript.

Hausotter, Jette (2010): „Zwischen Emanzipation und Einpassung: postfeministische Verwicklungen in Politik und Popkultur", in: Feministisches Institut Hamburg: Analysen, Positionen \& Beratung. [http://www.feministisches-institut.de/ wp-content/uploads/2010/10/Postfeministische_Verwicklungen.pdf; abgerufen am 21.03.2020].

Hoven, Elisa (2018): „Das neue Sexualstrafrecht - Der Prozess einer Reform“, in: Kriminalpolitische Zeitschrift 1/2018. [https://kripoz.de/2018/01/12/das-neuesexualstrafrecht-der-prozess-einer-reform/; abgerufen am 26.03.2020].

Jäger, Margarete (1999): „Ethnisierung von Sexismus im Einwanderungsdiskurs. Analyse einer Diskursverschränkung“. Vortrag, gehalten am 16.09.1999 an der Georg-August-Universität Göttingen beim Kolloquium: Wissenstransfer zwischen Experten und Laien. Umrisse einer Transferwissenschaft. [http://www. diss-duisburg.de/Internetbibliothek/Artikel/Ethnisierung_von_Sexismus.htm; abgerufen am 24.3.2020].

Kemper, Andreas (2014): Keimzelle der Nation. Familien- und Geschlechterpolitische Positionen der AfD. Eine Expertise. [http://library.fes.de/pdf-files/dialog/ 10641-20140414.pdf; abgerufen am 21.03.2020].

Keskinkılıç, Ozan (2016a): „,Der orientalische Mann“ vor|nach Köln. Zur sexuellkulturellen Dynamik des antimuslimischen Rassismus in der Fluchtdebatte“, in: Regina Römhild/Anja Schwanhäußer/Birgit zur Nieden/Göcze Yurdakul (Hg.), Witnessing the Transition: Moments in the Long Summer of Migration, Berlin: BIM, S. 61-73.

Kessl, Fabian (2011): „Pädagogisierungen - eine vernachlässigte Dimension in der Geschlechterforschung zur gegenwärtigen Transformation von Sozial-, Bildungs- und Erziehungspolitik“, in: Casale/Forster, Ungleiche Geschlechtergleichheit. Geschlechterpolitik und Theorien des Humankapitals, S. 61-75.

Kopke, Christoph (2017): „Verschwörungsmythen und Feindbilder in der AfD und in der neuen Protestbewegung von rechts“, in: NK - Neue Kriminalpolitik 29 (1), S. 49-61.

Lang, Juliane (2015): „Familie und Vaterland in der Krise. Der extrem rechte Diskurs um Gender", in: Hark/Villa, Anti-Genderismus. Sexualität und Geschlecht als Schauplätze politischer Auseinandersetzungen, S. 167-181. 
Lutz, Helma (2007): Vom Weltmarkt in den Privathaushalt. Die neuen Dienstmädchen im Zeitalter der Globalisierung, Opladen: Barbara Budrich.

Magni, Maura (2016): Antirassistischer Feminismus in Deutschland - Die feministische Debatte nach den Silvester-Übergriffen 2015 zwischen Neo-Orientalismus und Rassismuskritik. Bachelorarbeit, Berlin. [https:/www.academia.edu/ 33443690/Antirassistischer_Feminismus_in_Deutschland_Die_feministische_D ebatte_nach_den_Silvester-Übergriffen_2015_zwischen_Neo-Orientalismus und_Rassismuskritik; abgerufen am 26.03.2020].

Mayring, Philipp (2008): Qualitative Inhaltsanalyse. Grundlagen und Techniken, Weinheim/Basel: Beltz.

McRobbie, Angela (2010): Top Girls. Feminismus und der Aufstieg des neoliberalen Geschlechterregimes, Wiesbaden: Springer VS Verlag.

McRobbie, Angela (2011): „Beyond Post-Feminism“, in: Public Policy Research 18 (3), S. 179-184.

Mecheril, Paul (2002): „Natio-kulturelle Mitgliedschaft - ein Begriff und die Methode seiner Generierung“, in: Tertium comparationis 8 (2), S. 104-115. [https:// www.pedocs.de/volltexte/2011/2924/pdf/TC_2_2002_meche_D_A.pdf; abgerufen am 25.03.2020].

Mecheril, Paul (2017): „Flucht. Sex und Diskurse“, in: Christian Spatscheck/ Barbara Thiessen (Hg.), Inklusive und Soziale Arbeit. Teilhabe und Vielfalt als gesellschaftliche Gestaltungsfelder, Opladen: Barbara Budrich, S. 67-74.

Messerschmidt, Astrid (2016): „Nach Köln - sprechen über Sexismus und Rassismus“. Vortrag am 28. Januar 2016 in Tübingen bei einer Veranstaltung des Netzwerks für Rassismuskritische Migrationspädagogik in Baden-Württemberg. [https://www.rassismuskritik-bw.de/?ddownload=1014; abgerufen am 25.03. 2020].

Notz, Gisela (2015): Kritik des Familismus. Theorie und soziale Realität eines ideologischen Gemäldes, Stuttgart: Schmetterling Verlag.

Opratko, Benjamin (2019): „Autoritäre Wende, populistische Wette“, in: Ruth Daellenbach/Beat Ringger/Pascal Zwicky (Hg.), Reclaim Democracy. Demokratie stärken und weiterentwickeln, Zürich: Edition 8, S. 141-148.

Puar, Jasbir K (2007): Terrorist Assemblages. Homonationalism in Queer Times, Durham/London: Duke University Press.

Rams, Patricia (2017): „Zum antimuslimischen Rassismus im Dominanzfeminismus. Eine diskurstheoretisch fundierte frame-Analyse von Alice Schwarzers Beiträgen in ,Der Schock. Die Silvesternacht in Köln““, in: Forum Demokratieforschung. Beiträge aus Studium und Lehre. Working-Paper-Reihe im Fachgebiet Demokratieforschung am Institut für Politikwissenschaft der PhilippsUniversität Marburg. Working Paper Nr. 12. [https://www.uni-marburg.de/de/ $\mathrm{fb} 03 /$ politikwissenschaft/fachgebiete/brd/working-paper/workingpaper12.pdf; abgerufen am 21.03.2020]. 
Rendtorff, Barbara (2017): „Sexualisierungen als Elemente der Fremdabwehr“, in: Feministische Studien 35 (2), S. 302-317.

Rendtorff, Barbara/Riegraf, Birgit/Mahs, Claudia (2019): Struktur und Dynamik Un/Gleichzeitigkeiten im Geschlechterverhältnis, Wiesbaden: Springer VS.

Salgado, Rubia (2015): Aus der Praxis im Dissens, Bielefeld: transcript.

Scheele, Sebastian (2016): „Von Antifeminismus zu ,Anti-Genderismus“? Eine diskursive Verschiebung und ihre Hintergründe“. Keynote auf der Tagung „Gegner*innenaufklärung - Informationen und Analysen zu Anti-Feminismus“, Berlin: Gunda-Werner Institut in der Heinrich-Böll-Stiftung. [http://www.gwiboell.de/stes/default/files/uploads/2016/08/scheeleIdiskursiveIverschiebungIanti feminismus.pdf; abgerufen am 21.9.2018].

Scott, Joan (2007): Politics of the Veil, Princeton: University Press.

Soiland, Tove (2009): „Gender als Selbstmanagement. Zur Reprivatisierung des Geschlechts in der gegenwärtigen Gleichstellungspolitik“, in: Sünne Andresen/ Mechthild Koreuber/Dorothea Lüdke (Hg.), Gender und Diversity. Albtraum oder Traumpaar? Interdisziplinärer Dialog zur ,Modernisierung ' von Geschlechter- und Gleichstellungspolitik, Wiesbaden: VS Verlag für Sozialwissenschaften: S. 35-51.

Stojanov, Krassimir (2018): „Gemeinsame Wertebildung anstatt ,Wertevermittlung' an Migrant/innen“, in: ÖDaF-Mitteilungen 34 (1), S. 64-72.

Strasser, Sabine 2010: „Ist der Multikulturalismus noch zu retten?“, in: Sabine Strasser/Elisabeth Holzleithner (Hg.), Multikulturalismus queer gelesen. Zwangsheirat und gleichgeschlechtliche Ehe in pluralen Gesellschaften, Frankfurt/New York, S. 342-366.

Völker, Susanne 2016: „Geht es um Schutz? Verletzende Dynamiken: Sexualisierte Gewalt und rassistische Instrumentalisierungen“, in: blog feministische studien vom 21.02.2016. [http://blog.feministische-studien.de/2016/01/geht-es-umschutz-verletzende-dynamiken-sexualisierte-gewalt-und-rassistische-instrumentalisierungen/; abgerufen am 28.06.2016].

Witzel, Andreas (2000): „Das problemzentrierte Interview“, in: Forum Qualitative Sozialforschung 1 (1). [http://www.qualitative-research.net/index.php/fqs/rt/prin terFriendly/1132/2519; abgerufen am 13.05.2020 ].

\section{QuelLen}

\section{Textkorpus Diskursanalyse}

AfD (2017): Programm für Deutschland. Wahlprogramm der Alternative für Deutschland für die Wahl zum Deutschen Bundestag am 24. September 2017. Beschlossen auf dem Bundesparteitag in Köln am 22./23. April 2017. Berlin. 
[https://cdn.afd.tools/wp-content/uploads/sites/111/2017/06/2017-06-01_AfDBundestagswahlprogramm_Onlinefassung.pdf; abgerufen am 17.03.2020].

Balci, Güner (2016) „Das archaische Frauenbild bedroht uns alle.“ Interview, geführt von Andrea Seibel, in: welt.de vom 13.01.2016. [https://www.welt.de/ politik/deutschland/article150929502/Das-archaische-Frauenbild-bedroht-unsalle.htm; abgerufen am 18.03.2020].

Bilge, Leyla (2018): „Der Islam in unserer Gesellschaft“. Rede im Kloster Fürstenfeld am 13.07.2018. [https://www.youtube.com/watch?v=r-eXFFfpWmU; abgerufen am 18.03.2020].

Buschkowsky, Heinz (2015): „Buschkowsky befürchtet Streit: ,Im Griff haben wir die Situation nicht““. Interview, geführt von Christian Wilp, in: ntv vom 01.10. 2015. [https://www.n-tv.de/politik/Im-Griff-haben-wir-die-Situation-nicht-arti cle16052261.html; abgerufen am 18.03.2020].

Daoud, Kamel (2016a): „Islam und Körper. Das sexuelle Elend der arabischen Welt", in: faz.net vom 18.02.2016. [https://www.faz.net/aktuell/feuilleton/islam -und-koerper-das-sexuelle-elend-der-arabischen-welt-14075502.html; abgerufen am 18.03.2020].

Daoud, Kamel (2016b): „Als deutscher Rentner hätte ich Angst.“ Interview, geführt von Georg Blume, in: zeit.de vom 03.03.2016. [http://www.zeit.de/2016/11/ kamel-daoud-schriftsteller-algerien-islamkritik; abgerufen am 18.03.2020].

Elsässer, Jürgen (2017): „Italienische Forscher bestätigen ,biologisches Aussterben“ der Europäer - ,Austausch der Nationen“ ist in vollem Gange“, in: Compact Online vom 08.08.2017. [https://www.compact-online.de/italienische-forscherbestaetigen-biologisches-aussterben-der-europaeer-austausch-der-nationen-istin-vollem-gange/; abgerufen am 17.03.2020].

Emma (2015): Interview mit Julia Glöckner. [https://www.emma.de/lesesaal/60750 \#pages/25; abgerufen am 17.03.2020].

Erk, Daniel (2019): „Identitäre Bewegung. Sind sie jetzt am Ende?“, n: Zeit Online vom 08.04.2019. [https://www.zeit.de/2019/15/identitaere-bewegung-fpoerechtsextremismus-christchurch-anschlag; abgerufen am 07.04.2020].

Fachstelle Gender, GMF und Rechtsextremismus (2017): „,Pegida“ und Gender: Von der ,Homo-Lobby“, ,Umvolkung“ und ,Gender-Tanten““, in: Belltower News - Netz für digitale Zivilgesellschaft vom 21.06.2017. [https://www. belltower.news/pegida-und-gender-von-der-homolobby-umvolkung-und-gender tanten-44224/; abgerufen am 17.03.2020].

focus (2018): „Gehört der Islam zu Deutschland? Heinz Buschkowsky unterstützt Seehofers Islam-Aussage“, in: focus.de vom 20.03.2018. [https://www.focus.de/ politik/deutschland/gehoert-der-islam-zu-deutschland-csu-chef-seehofer-be kommt-fuer-umstrittene-aeusserung-unterstuetzung-aus-der-spd_id_8625251. html; abgerufen am 18.03.2020].

Free-Gender (2011): Raus aus den Köpfen - Genderterror abschaffen. Zitiert nach: Diskursatlas Antifeminismus (o.J.) [http://www.diskursatlas.de/index.php?title= 
Keimzelle_der_Nation; abgerufen am 18.03.2020]. (Die Webseite www.freegender.de selbst ist nicht mehr aufrufbar).

Greiner, Regine (2015): „Potsdam/Gymnasium Michendorf: Keine Miniröcke wegen Flüchtlingen“, in: maz-online.de vom 30.06.2015. [https://www.mazonline.de/Lokales/Potsdam/Keine-Miniroecke-wegen-Fluechtlingen; abgerufen am 18.03.2020].

Höcke, Björn (2016): (Facebook-Eintrag, ohne Titel) [https://www.facebook.com/ Bjoern.Hoecke.AfD/photos/a.1424703574437591/1650192565222023/?type= 3\&theater; abgerufen am 18.03.2020].

Identitäre Bewegung (2019): Vortragsabend in Wien, 19.05.2019. [https://www. identitaere-bewegung.at/vortragsabend-in-wien/; abgerufen am 09.04.2020].

Krüger, Karen (2016): „Geschlecht und Islam: Lassen Sie uns über Sex reden“, in: faz.net vom 23.01.2016. [https://www.faz.net/aktuell/feuilleton/geschlecht-undislam-lassen-sie-uns-ueber-sex-reden-14029390-p4.html; abgerufen am 18.03. 2020].

Lämmel, Andreas/Vaatz, Arnold (2016): Mal ernsthaft: Mit Gender-Gaga gegen das arabische Frauenbild? (Veranstaltungsplakat) [https://www.arnold-vaatz.de/ mal-ernsthaft-mit-gendergaga-gegen-das-arabische-frauenbild-wie-ideologienunsere-freiheiten-bed; abgerufen am 18.03.2020].

Palmer, Boris (2015): „Boris Palmer zu Flüchtlingen: ,Können Asylstandards nicht halten ““. Interview, geführt von Anja Maier, in: Die Tageszeitung (taz) vom 20.09.2015. [https://taz.de/Boris-Palmer-zu-Fluechtlingen/!5234269/; abgerufen am 18.03.2020].

PI-News (2017): „Europas kinderlose Politiker“, in: PI-News vom 19.05.2017. [http://www.pi-news.net/2017/05/europas-kinderlose-politiker/; abgerufen am 17.03.2020].

Poggenburg, André (2018): „Völlig richtig, die \#meetoo-Kampagne ist zu einer reinen Farce verkommen." Tweet vom 13.01.2018. [https://twitter.com/poggen burgandre/status/952353929538437120?lang=de; abgerufen am 18.03.2020].

Presseportal der AfD (2016): „Petry: Massenhafter Mißbrauch von Frauen in Köln erinnert an rechtlose Zustände zum Kriegsende“, in: Presseportal vom 05.01. 2016. [https://www.presseportal.de/pm/110332/3217652; abgerufen am 18.03. 2020].

Rabeder, René (2019): „Mädls, wacht endlich auf! Der edle Wilde ist eine Illusion“, in: Wochenblick vom 14.01.2019. [https://www.wochenblick.at/maedls-wachtendlich-auf-der-edle-wilde-ist-eine-illusion/; abgerufen am 18.03.2020].

Ramadani, Zana (2016): „Seid wütend auf die muslimischen Frauen!“ Interview, geführt von Kathrin Spoerr, in: welt.de vom 14.01.2016. [http://www.welt.de/ vermischtes/article150989935/Seid-wuetend-auf-die-muslimischen-Frauen. html; abgerufen am 18.03.2020].

rp online (2016): „Die Übergriffe von Köln und die Folgen. Kaum einer hat sich Gedanken gemacht, wer da ins Land kommt“, in: rp online vom 16.01.2016. 
[https://rp-online.de/nrw/staedte/koeln/gastbeitrag-zu-koeln-und-den-folgen-esgibt-kein-schluessiges-konzept-fuer-integration_aid-21223975; abgerufen am 18.03.2020].

Schirmbeck, Samuel (2016): „Muslimisches Frauenbild. Sie hassen uns“, in: faz.net vom 06.07.2016. [http://www.faz.net/aktuell/politik/inland/gastbeitrag-von-sa muel-schirmbeck-zum-muslimischen-frauenbild-14007010.html; abgerufen am 18.03.2020].

Schröder, Kristina (2016): „Und es hat doch mit dem Islam zu tun“, in: Cicero vom 16.08.2016. [https://www.cicero.de/innenpolitik/integrationsdebatte-und-es-hatdoch-mit-dem-islam-zu-tun; abgerufen am 18.03.2020].

Schwarzer, Alice (2016) „Kalaschnikows, Sprenggürtel und jetzt die sexuelle Gewalt“. Interview, geführt von Rolf Poschardt, in: welt.de vom 15.01.2016. [http: //www.welt.de/politik/deutschland/article151065691/Kalaschnikows-Spreng guertel-und-jetzt-die-sexuelle-Gewalt.html; abgerufen am 18.03.2020].

Sezession (2015): „Migration und sexuelle Gewalt: Anpassungsstrategien an einem deutschen Gymnasium“, in: Sezession vom 25.06.2015. [https://sezession.de/ 50234/migration-und-sexuelle-gewalt-anpassungsstrategien-an-einem-deut schen-gymnasium; abgerufen am 26.03.2020].

Spahn, Jens (2014): „Kein Rabatt für rassistische Migranten!“, in: welt.de vom 26.07.2014. [https://www.welt.de/debatte/kommentare/article130572127/KeinRabatt-fuer-rassistische-Migranten.html; abgerufen am 18.03.2020].

Zukunft Heimat (o.J.): [https://zukunft-heimat.org/; abgerufen am 17.03.2020].

\section{Weitere Quellen}

Herman, Eva (2006): Das Eva-Prinzip: Für eine neue Weiblichkeit, München: Verlag Pendo.

Pirinçci, Akif (2014): Attacke auf den Mainstream - ,Deutschland von Sinnen ' und die Medien. Hg. von Andreas Lombard, Waltrop: Edition Sonderwege, Manuscriptum Verlagsbuchhandlung.

Sarrazin, Thilo (2010): Deutschland schafft sich ab. Wie wir unser Land aufs Spiel setzen, München: DVA.

\section{Zitierte Lehrbücher}

Buchwald-Wargenau, Isabel (2017): Mein Leben in Deutschland - der Orientierungskurs. Kursbuch Basiswissen Politik, Geschichte, Gesellschaft, München: Hueber Verlag.

Butler, Ellen/Kotas, Ondrej/Sturm, Marti/Sum, Barbara/Wolf, Nita Esther/Würtz, Helga (2017): 100 Stunden Deutschland. Kurs- und Übungsbuch mit Audios online. Orientierungskurs Politik, Geschichte, Kultur, Stuttgart: Klett Sprachen. 
Gaidosch, Ulrike/Müller, Christine (2017): Zur Orientierung. Basiswissen Deutschland. Für Orientierungskurse mit 100 Unterrichtseinheiten. 7. Aufl., München: Hueber Verlag.

Kilimann, Angela/Kotas, Ondrej/Schiffhauer, Ina/Skrodzki, Johanna (2013): 60 Stunden Deutschland. Orientierungskurs Politik - Geschichte - Kultur. Kursund Übungsbuch + Audio-CD, Stuttgart: Klett Sprachen.

Schote, Joachim (2017): Orientierungskurs. Aktuelle Ausgabe A2/B1. Grundwissen Politik, Geschichte und Gesellschaft in Deutschland, Berlin: Cornelsen Verlag.

\section{Interviews und Teilnehmende Beobachtungen}

Ort1/KL: Interview mit der Kursleitung in Ort 1, geführt am 25.5.2018. Ort1/TN1: Interview Teilnehmerin 1 in Ort 1, geführt am 23.5.2018. Ort1/TN2: Interview Teilnehmerin 2 in Ort 1, geführt am 24.5.2018. Ort1/TN3: Interview Teilnehmer 3 in Ort 1, geführt am 25.5.2018. Ort1/TN4: Interview Teilnehmer 4 in Ort1, geführt am 25.5.2018. Ort1/TB: Teilnehmende Beobachtung im Kurs in Ort 1 (23./24.5.2018).

Ort2/KL: Interview mit der Kursleitung von Kurs 1 in Ort 2, geführt am 04.06. 2018.

Ort2/OE: Interview Organisationsebene in Ort 2, geführt am 04.06.2018.

Ort2/TN1: Interview Teilnehmerin 1 (Kurs 1) in Ort 2, geführt am 04.06.2018.

Ort2/TN2: Interview Teilnehmer 2 (Kurs 1) in Ort 2, geführt am 05.06.2018.

Ort2/TN3: Interview Teilnehmer 3 (Kurs 2) in Ort 2, geführt am 06.06.2018.

Ort2/TN4: Interview Teilnehmer 4 (Kurs 2) in Ort 2, geführt am 6.6.2018.

Ort2/TB1: Teilnehmende Beobachtung in Kurs I in Ort 2 (04./05.06.2018).

Ort2/TB2: Teilnehmende Beobachtung in Kurs II in Ort 2 (05.06.2018).

Ort3/KL1: Interview mit Kursleitung1 in Ort 3, geführt am 18.06.2018. Ort3/OE: Interview Organisationsebene in Ort 3, geführt am 20.06.2018. Ort3/TN1: Interview Teilnehmer 1 in Ort 3, geführt am19.06.2018. Ort3/TN2: Interview Teilnehmerin 2 in Ort 3, geführt am 19.06.2018. Ort3/TN3: Interview Teilnehmer 3 in Ort 3, geführt am 19.06.2018. Ort3/TB1: Teilnehmende Beobachtung in Ort 3 (18.06.2018).

Ort4/KL: Interview mit der Kursleitung in Ort 4, geführt am 24.04.2018. Ort4/TN1: Interview Teilnehmer1 und 2 in Ort 4, geführt am 24.04.2018. Ort4/TN2: Interview Teilnehmerin 3 in Ort 4, geführt am 25.04.2018. Ort4/TB1: teilnehmende Beobachtung1 in Kurs I in Ort 4 (23.04.2018). Ort4/TB2: teilnehmende Beobachtung2 in Kurs I in Ort 4 (24.04.2018).

Ort5/KL1: Interview mit der Kursleitung1 in Ort 5, geführt am 17.07.2018. 
Ort5/KL2: Interview mit der Kursleitung2 in Ort 5, geführt am 17.07.2018.

Ort5/OE: Interview Organisationsebene in Ort 5, geführt am 16.07.2018.

Ort5/TN-Gruppe: Gruppengespräch mit 8 Teilnehmer_innen in Kurs II in Ort 5, geführt am 17.07.2018.

Ort5/TN1: Interview mit Teilnehmer1 in Ort 5, geführt am 16.07.2018.

Ort5/TN2: Interview mit Teilnehmerin2 in Ort 5, geführt am 16.07.2018.

Ort5/TB1: teilnehmende Beobachtung in Kurs I in Ort 5 (16.07.2018).

Ort5/TB2: teilnehmende Beobachtung in Kurs II in Ort 5 (17.07.2018).

Ort6/KL1: Interview mit Kursleitung1 in Ort 6, geführt am 02.05.2018.

Ort6/KL2: Interview mit Kursleitung2 in Ort 6, geführt am 02.05.2018.

Ort6/KL3: Interview mit Kursleitung3 in Ort 6, geführt am 03.05.2018.

Ort6/OE: Interview Organisationsebene in Ort 6, geführt am 02.05.2018.

Ort6/Gruppengespräch mit 7 Teilnehmer_innen und der Kursleitung (Kurs1) in Ort 6, geführt am 03.05.2018.

Ort6/TB1: teilnehmende Beobachtung in Kurs I in Ort 6 (02.05.2018/03.05.2018).

Ort6/TB2: teilnehmende Beobachtung in Kurs II in Ort 6 (03.05.2018).

Ort7/KL: Interview KL1 mit der Kursleitung in Ort 7, geführt am 21.06.2018.

Ort7/TN1: Interview mit Teilnehmer1 in Ort 7, geführt am 21.06.2018.

Ort7/TN2: Interview mit Teilnehmerin2 in Ort 7, geführt am 21.06.2018.

Ort7/TN3: Interview mit Teilnehmerin2 in Ort 7, geführt am 21.06.2018.

Ort7/TB: teilnehmende Beobachtung in Kurs I in Ort 7 (21.06.2018). 
